\title{
ENTIRE SPACELIKE HYPERSURFACES OF CONSTANT GAUSS CURVATURE IN MINKOWSKI SPACE
}

\author{
PIERRE BAYARD AND OLIVER C. SCHNÜRER
}

\begin{abstract}
We prove existence and stability of smooth entire strictly convex spacelike hypersurfaces of prescribed Gauß curvature in Minkowski space. The proof is based on barrier constructions and local a priori estimates.
\end{abstract}

\section{INTRODUCTION}

In Minkowski space $\mathbb{L}^{n+1}$, the Gauß curvature of graph $u, u: \mathbb{R}^{n} \rightarrow \mathbb{R}$, is given by

$$
K[u]=\frac{\operatorname{det} D^{2} u}{\left(1-|D u|^{2}\right)^{\frac{n+2}{2}}} .
$$

We consider that equation for strictly convex strictly spacelike functions $u$ (see Section 2 for definitions). The Gauß map sends every point of the hypersurface graph $u$ to its future directed unit normal which is a point in hyperbolic space $\left\{x \in \mathbb{L}^{n+1}:\langle x, x\rangle=-1, x^{n+1}>0\right\}$. In this paper we study convex spacelike hypersurfaces of constant Gauß curvature with prescribed Gauß map image. We solve the corresponding fully nonlinear elliptic partial differential equation on a sequence of growing balls and pass to a limit. In addition, we study logarithmic Gauß curvature flow for convex spacelike hypersurfaces with given Gauß map image. These solutions converge to solutions of the equation of constant Gauß curvature. Thus the solutions to the elliptic equation are dynamically stable.

The construction of hypersurfaces of prescribed Gauß curvature and of solutions to logarithmic Gauß curvature flow uses barriers. For these barriers, we have the following existence result. For details, we refer to Theorem 4.3.

Theorem 1.1. There exist spacelike (viscosity) sub- and supersolutions $\underline{u}$ and $\bar{u}$ to the equation of prescribed constant positive Gauß curvature which are at infinity close to $V_{F}$, where $V_{F}(x):=\sup _{\lambda \in F} x \cdot \lambda$ and $F$ is the closure of

Date: December 2006.

Key words and phrases. Gauß curvature, Minkowski space, stability, regularity, spacelike, Gauß map image. 
some open non-empty subset of the ideal boundary $S^{n-1}$ of hyperbolic space with $\partial F \in C^{1,1}$. Moreover, the subsolution $\underline{u}$ is convex.

Our main results are the existence results for solutions to the equation of prescribed Gauß curvature and for solutions to logarithmic Gauß curvature flow. The result for the equation of prescribed Gauß curvature is

Theorem 1.2. Let $\underline{u} \leq \bar{u}$ be barriers as above with $K[\underline{u}]>1>K[\bar{u}]$ in the viscosity sense, close to $V_{F}$ at infinity. Then there exists a unique smooth strictly convex strictly spacelike function $u: \mathbb{R}^{n} \rightarrow \mathbb{R}$ with $\underline{u} \leq u \leq \bar{u}$ which solves the equation of prescribed Gauß curvature one

$$
K[u]=\frac{\operatorname{det} D^{2} u}{\left(1-|D u|^{2}\right)^{\frac{n+2}{2}}}=1 \quad \text { in } \mathbb{R}^{n},
$$

i.e. graph $u \subset \mathbb{L}^{n+1}$ is a strictly convex strictly spacelike hypersurface of Gauß curvature one. Moreover, the image of the Gauß map is the hyperbolic space convex hull of $F$.

For logarithmic Gauß curvature flow, we have

Theorem 1.3. Let $\underline{u}$ and $\bar{u}$ be barriers as in Theorem 1.2. Let $u_{0}: \mathbb{R}^{n} \rightarrow \mathbb{R}$ be a smooth strictly convex strictly spacelike function with $\underline{u} \leq u_{0} \leq \bar{u}$ such that $\log K\left[u_{0}\right]$ is uniformly bounded. Then there exists a unique strictly convex strictly spacelike function $u: C^{\infty}\left(\mathbb{R}^{n} \times(0, \infty)\right) \cap C^{0}\left(\mathbb{R}^{n} \times[0, \infty)\right)$ solving

$$
\begin{cases}\dot{u}=\sqrt{1-|D u|^{2}} \cdot \log K[u] & \text { in } \mathbb{R}^{n} \times(0, \infty), \\ u(\cdot, 0)=u_{0} & \text { in } \mathbb{R}^{n}, \\ \underline{u} \leq u(\cdot, t) \leq \bar{u} & \text { in } \mathbb{R}^{n} \text { for all } t \geq 0\end{cases}
$$

such that $\log K[u(\cdot, t)]$ is uniformly bounded for all $t \geq 0$. Moreover, as $t \rightarrow$ $\infty$, the functions $u(\cdot, t)$ converge exponentially to the solution in Theorem 1.2.

The barriers for Theorem 1.1/4.3 are obtained as follows. We apply a Lorentz transformation to a "semitrough", a hypersurface of constant Gauß curvature, which has Gauß map image equal to half the hyperbolic space. Then we take suprema and infima over such semitroughs and obtain barriers $\underline{u}$ and $\bar{u}$. It is essential for the following to control the behavior of these barriers near infinity during this construction.

In order to prove Theorem 1.2 and Theorem 1.3, we solve (1.1) and an equation similar to (1.2) between the barriers $\underline{u}$ and $\bar{u}$ on balls with additional Dirichlet boundary conditions imposed. We do such on a sequence of growing balls. For these auxiliary solutions, we prove a priori estimates for the first and second derivatives which are local in space and uniform in time. For proving $C^{1}$-estimates it is necessary to have barriers which are close to 
each other at spatial infinity. Then $C^{2}$-estimates require only control of the $C^{0}$-behavior of $u$. These a priori estimates allow to extract subsequences converging to the desired solutions.

Many of our techniques extend to Euclidean space and to the situation where $f=f(X, \nu, t)$.

Theorem 1.3 shows that the solutions found in Theorem 1.2 are dynamically stable. In the flow equation, the logarithm is useful to preserve convexity. The flow equation $\dot{u}=\log K$ is a less geometric alternative for which analogous results can be proved by methods similar to the ones used here.

In our case, the stability issue follows directly from the evolution equation of the normal velocity. In order to guarantee convergence to the elliptic solution, we have to impose that solutions are $C^{0}$-close at infinity to the elliptic solution $\tilde{u}$. Otherwise, $u$ might converge to $\tilde{u}+c$ instead. Thus it is not too restrictive to start between these barriers. Of course, the barriers are also crucial for proving local $C^{1}$ a priori estimates.

Note that (1.2) describes hypersurfaces moving with normal velocity equal to the logarithm of the Gauß curvature $K, \frac{d}{d t} X=\log K \nu$. By scaling graph $u$, we can use Theorem 1.2 to find convex spacelike hypersurfaces of constant Gauß curvature $f_{0}>0$. Replacing the flow equation (1.2) in Theorem 1.3 by

$$
\begin{aligned}
\dot{u} & =\sqrt{1-|D u|^{2}} \cdot\left(\log K[u]-\log f_{0}\right) \\
& \equiv \sqrt{1-|D u|^{2}} \cdot\left(\log \frac{\operatorname{det} D^{2} u}{\left(1-|D u|^{2}\right)^{\frac{n+2}{2}}}-\log f_{0}\right)
\end{aligned}
$$

and using barriers with $K[\underline{u}]>f_{0}>K[\bar{u}], \lim _{t \rightarrow \infty} u(\cdot, t)$ converges to the hypersurface of Gauß curvature $f_{0}$ mentioned above.

Let us quote some results concerning hypersurfaces of prescribed mean and Gauß curvature in Minkowski space.

In [20], Andrejs Treibergs classifies all the entire spacelike hypersurfaces of constant mean curvature in $\mathbb{L}^{n+1}$ by their boundary values at infinity, and in [5], Hyeong In Choi and Andrejs Treibergs describe the Gauß maps of the entire spacelike constant mean curvature hypersurfaces in $\mathbb{L}^{n+1}$; they prove the following: for any closed set in the ideal boundary at infinity of the hyperbolic space which has more than two points, there exists an entire spacelike hypersurface with constant mean curvature whose Gauß map image is the hyperbolic space convex hull of the set.

The Dirichlet problem for the prescribed Gauß curvature equation in Minkowski space is solved on convex domains by Philippe Delanoë in [7]. Bo 
Guan solved the problem in [14] under the weaker assumption of the existence of a lower barrier. In [17], An-Min Li proved the existence of entire convex spacelike hypersurfaces of prescribed positive Gauß curvature which stay at a bounded distance of a light-cone.

In [15], Bo Guan, Huai-Yu Jian, and Richard Schoen prove the following: for every closed set in the ideal boundary at infinity of the hyperbolic space which is not contained in any hyperplane, there exists a Lipschitz hypersurface whose graph solves the prescribed constant Gauß curvature equation in a weak sense and whose Gauß map image is the convex hull of the set. Moreover, they study a Minkowski type problem on half of hyperbolic space.

Our results are similar to a result by Bo Guan, Huai-Yu Jian, and Richard Schoen [15, Theorem 3.5]. Our a priori estimates, especially the local $C^{1}$ estimates, do not degenerate when we solve auxiliary problems on a sequence of growing balls. Thus the limit of the solutions to our auxiliary problems is smooth and strictly spacelike.

Theorem 1.3 allows to deform entire hypersurfaces by a fully nonlinear flow equation. Before, such has been done for mean curvature flow by Klaus Ecker and Gerhard Huisken in Euclidean space [9] and by Klaus Ecker in Minkowski space [8]. There is a mean curvature flow approach by Mark Aarons [1] to find entire spacelike hypersurfaces of constant mean curvature as classified by Andrejs Treibergs in [20]. Stability of non-compact solutions to geometric flow equations was studied by the second author and Albert Chau [4] for Kähler-Ricci flow and for mean curvature flow with Julie Clutterbuck and Felix Schulze [6].

Ricci flow of non-compact manifolds has been considered by Wan-Xiong Shi [19]. He solves the initial value problem for metrics of bounded curvature. Similarly, we assume initially bounded Gauß curvature in Theorem 1.3. Note that for Kähler manifolds, Ricci flow can be rewritten as $\dot{u}=\log \operatorname{det} u_{i \bar{\jmath}}$ for the Kähler potential.

The rest of the paper is organized as follows: In Section 2 we introduce some terminology. We study the Gauß map and construct barriers in Sections 3 and 4. Local $C^{1}$ - and $C^{2}$-estimates are derived in Sections 5 and 6 . We obtain the existence and convergence results mentioned above in Section 7 . We mention non-compact comparison principles in A. In Appendix B, we solve auxiliary problems on balls and prove a local normal velocity bound in Appendix $\mathrm{C}$ that allows to relax the uniform initial normal velocity bound. A technical lemma in Section D finishes the paper.

Acknowledgement: The first author was supported by SFB 647 during his visit in Berlin in December 2006. 


\section{Definitions and Notation}

We say that a function $u: \Omega \rightarrow \mathbb{R}$ is strictly convex, if its Hessian $D^{2} u=$ $\left(u_{i j}\right)$ has positive eigenvalues. We say that such a function is uniformly strictly convex, if the eigenvalues of $D^{2} u$ on $\Omega$ are uniformly bounded below by a positive constant. A function $u: \Omega \times[0, \infty)$ is said to be (uniformly) strictly convex, if $u(\cdot, t)$ is (uniformly) strictly convex for each $t$ and, in the uniformly strictly convex case, if the positive lower bound on the eigenvalues of the Hessian is independent of $t$.

A function $u: \Omega \rightarrow \mathbb{R}$ is called strictly spacelike, if graph $u$ is strictly spacelike, i.e. if $|D u|<1$. Such a function is uniformly strictly spacelike, if $\sup _{\Omega}|D u|<1$. We say that a Lipschitz function $u$ is strictly spacelike in a set $\Omega$, if there exists some $\vartheta>0$ such that $|u(x)-u(y)| \leq(1-\vartheta)|x-y|$ for all $x, y \in \Omega$. Similar to the definition of convexity, we say that $u: \Omega \times[0, \infty)$ is (uniformly) strictly spacelike, if $u(\cdot, t)$ has this property for any $t$.

A function $f$ is called uniformly positive, if it is bounded below by a uniform positive constant everywhere on its domain of definition.

2.1. Notation. We say that a function $u$ solving a parabolic equation is in $C^{2}$, if $u(\cdot, t)$ is in $C^{2}$ for every $t$. The space $C^{2,1}$ denotes those functions, where in addition all first derivatives are continuous.

We use Greek indices running from 1 to $n+1$ for tensors in $(n+1)$ dimensional Minkowski space. Latin indices refer to quantities on spacelike hypersurfaces and run from 1 to $n$. The Einstein summation convention is used to sum over pairs of upper and lower indices. We raise and lower indices of tensors with the respective metrics or its inverses. An exception is the Latin subscript $t$, we define $f_{t}=\frac{\partial f}{\partial t}$. We set $\dot{u}=\frac{\partial u}{\partial t}$.

We use $\mathbb{L}^{n+1}$ to denote $(n+1)$-dimensional Minkowski space with its metric $\left(\bar{g}_{\alpha \beta}\right)=\operatorname{diag}(1, \ldots, 1,-1)$. We agree to always use coordinate systems in Minkowski space such that the metric has this form. Therefore the Codazzi equations imply that the first covariant derivative of the second fundamental form is completely symmetric. We use $X=X(x, t)$ to denote the embedding vector of a manifold $M_{t}$ into $\mathbb{L}^{n+1}$ and $\frac{d}{d t} X=\dot{X}$ for its total time derivative. It is convenient to identify $M_{t}$ and its embedding in $\mathbb{L}^{n+1}$. An embedding induces a metric $\left(g_{i j}\right)$. We will consider strictly spacelike hypersurfaces $M_{t} \subset$ $\mathbb{L}^{n+1}$. If $M_{t}$ is locally represented as graph $u, u: \Omega \rightarrow \mathbb{R}, \Omega \subset \mathbb{R}^{n}$, being strictly spacelike is equivalent to $|D u|<1$, where $|D u|$ denotes the Euclidean norm of the gradient of $u$. Let us use $u_{i}$ to denote partial derivatives of $u$. Using the Kronecker delta $\delta^{*}$, we have $u_{i} \delta^{i j} u_{j} \equiv u_{i} u^{i}=|D u|^{2}$. The induced 
metric $\left(g_{i j}\right)$ of graph $u$ and its inverse $\left(g^{i j}\right)$ are given by

$$
g_{i j}=\delta_{i j}-u_{i} u_{j} \quad \text { and } \quad g^{i j}=\delta^{i j}+\frac{u^{i} u^{j}}{1-|D u|^{2}},
$$

respectively.

We choose $\left(\nu^{\alpha}\right)$ to be the future directed unit normal vector to $M_{t}$. If $M_{t}$ is locally represented as graph $u$, we get

$$
\begin{aligned}
\nu^{\alpha} & =\frac{(D u, 1)}{\sqrt{1-|D u|^{2}}} \\
\nu_{\alpha} & \equiv \bar{g}_{\alpha \beta} \nu^{\beta}=\frac{(D u,-1)}{\sqrt{1-|D u|^{2}}} .
\end{aligned}
$$

The embedding also induces a second fundamental form $\left(h_{i j}\right)$. In the graphical setting it is given in terms of partial derivatives by $h_{i j}=u_{i j} / \sqrt{1-|D u|^{2}}$. We denote its inverse by $\tilde{h}^{i j}$.

We write indices, preceded by semi-colons, e.g. $h_{i j ; k}$, to indicate covariant differentiation with respect to the induced metric. For derivatives in $\mathbb{L}^{n+1}$, we use expressions like $f_{\alpha}$. Setting $X_{; i j}^{\alpha}:=X_{, i j}^{\alpha}-\Gamma_{i j}^{k} X_{k}^{\alpha}$, where a colon indicates partial derivatives, the Gauß formula is

$$
X_{; i j}^{\alpha}=h_{i j} \nu^{\alpha}
$$

and the Weingarten equation is

$$
\nu_{; i}^{\alpha}=h_{i}^{k} X_{k}^{\alpha} \equiv h_{i l} g^{l k} X_{k}^{\alpha} .
$$

The eigenvalues of $h_{i j}$ with respect to $g_{i j}$ are the principal curvatures of the hypersurface and are denoted by $\lambda_{1}, \ldots, \lambda_{n}$. A hypersurface is called strictly convex, if all principal curvatures are strictly positive.

The Gauß curvature is the product of the principal curvatures

$$
K=\lambda_{1} \cdots \lambda_{n}=\frac{\operatorname{det} h_{i j}}{\operatorname{det} g_{i j}}
$$

For graph $u$, it is given by $K[u]:=\operatorname{det} u_{i j} \cdot\left(1-|D u|^{2}\right)^{-\frac{n+2}{2}}$, so the evolution equation

$$
\frac{d}{d t} X^{\alpha}=\dot{X}^{\alpha}=(\log K-\log f) \nu^{\alpha} \equiv(F-\hat{f}) \nu^{\alpha}
$$

can be rewritten for graphs as

$$
\dot{u}=\sqrt{1-|D u|^{2}}\left(\log \frac{\operatorname{det} D^{2} u}{\left(1-|D u|^{2}\right)^{\frac{n+2}{2}}}-\log f(x, u, t)\right)
$$

and is therefore parabolic precisely when $u$ is strictly convex and strictly spacelike. 
Let us also define the mean curvature $H=h_{i j} g^{i j}=\lambda_{1}+\cdots+\lambda_{n}$ and the squared norm of the second fundamental form $|A|^{2}=h_{i j} h_{k l} g^{i k} g^{j l}=$ $\lambda_{1}^{2}+\cdots+\lambda_{n}^{2}$.

It is often convenient to choose coordinate systems such that the metric tensor equals the Kronecker delta, $g_{i j}=\delta_{i j}$, and $\left(h_{i j}\right)$ is diagonal, $\left(h_{i j}\right)=$ $\operatorname{diag}\left(\lambda_{1}, \ldots, \lambda_{n}\right)$. Note that in such a coordinate system $F^{i j}:=\frac{\partial F\left(h_{i j}, g_{i j}\right)}{\partial h_{i j}}=$ $\tilde{h}^{i j}$ is diagonal.

For tensors $A$ and $B, A_{i j} \geq B_{i j}$ means that $\left(A_{i j}-B_{i j}\right)$ is positive definite. Finally, we use $c$ to denote universal, estimated constants.

In order to compute evolution equations, we use the Gauß equation and the Ricci identity for the second fundamental form

$$
\begin{aligned}
R_{i j k l} & =-h_{i k} h_{j l}+h_{i l} h_{j k}, \\
h_{i k ; l j} & =h_{i k ; j l}+h_{k}^{a} R_{a i l j}+h_{i}^{a} R_{a k l j} .
\end{aligned}
$$

2.2. Evolution Equations. Recall, see e. g. [10, 11], that for a hypersurface moving according to

$$
\frac{d}{d t} X^{\alpha}=(\log K-\log f(X, t)) \nu^{\alpha} \equiv\left(F\left(h_{i j}, g_{i j}\right)-\hat{f}(X, t)\right) \nu^{\alpha},
$$

we have

$$
\begin{aligned}
& \frac{d}{d t} g_{i j}=2(F-\hat{f}) h_{i j} \\
& \frac{d}{d t} h_{i j}=(F-\hat{f})_{; i j}+(F-\hat{f}) h_{i}^{k} h_{k j} \\
& \frac{d}{d t} h_{i}^{j}=(F-\hat{f})_{; i}^{j}-(F-\hat{f}) h_{i}^{k} h_{k}^{j}, \\
& \frac{d}{d t} \nu^{\alpha}=g^{i j}(F-\hat{f})_{; i} X_{; j}^{\alpha}, \\
& \frac{d}{d t}(F-\hat{f})= F^{i j}(F-\hat{f})_{; i j}-(F-\hat{f}) F^{i j} h_{i}^{k} h_{k j} \\
&-\hat{f}_{\alpha} \nu^{\alpha}(F-\hat{f})-\hat{f}_{t} \\
&= F^{i j}(F-\hat{f})_{; i j}-(F-\hat{f}) H-\hat{f}_{\alpha} \nu^{\alpha}(F-\hat{f})-\hat{f}_{t} .
\end{aligned}
$$

We define $\eta_{\alpha}=(0, \ldots, 0,1)$ and $\tilde{v}=\eta_{\alpha} \nu^{\alpha}$. The evolution equation for $\tilde{v}$ is given by

$$
\frac{d}{d t} \tilde{v}-F^{i j} \tilde{v}_{; i j}=-\tilde{v} H-\eta_{\alpha} X_{; i}^{\alpha} g^{i j} X_{; j}^{\beta} \hat{f}_{\beta}
$$




\section{The Gauss Map of an Entire Spacelike Hypersurface of Constant Gauss Curvature}

We recall here some results from $[5,15,20]$ concerning the Gauß map of an entire spacelike hypersurface of constant Gauß curvature in $\mathbb{L}^{n+1}$. Following $[20$, Section 6$]$, the blow down $V_{u}: \mathbb{R}^{n} \rightarrow \mathbb{R}$ of a convex (spacelike) function $u$ is defined by

$$
V_{u}(x)=\lim _{r \rightarrow \infty} \frac{u(r x)}{r} .
$$

Denoting by $Q$ the set of the convex homogeneous of degree one functions whose gradient has norm one whenever defined, the following holds (see [20, Theorem 1] for the prescribed constant mean curvature equation):

Lemma 3.1. For every admissible solution $u$ of the prescribed constant Gauß curvature equation (1.1) the blow down $V_{u}$ belongs to $Q$.

Proof. $V_{u}$ is clearly convex homogeneous of degree one. To prove that its gradient has norm one whenever defined, we just observe that the barrier construction of Treibergs for the prescribed constant mean curvature equation can be used for the prescribed constant Gauß curvature equation as well (see [20] page 52, step 1 in the proof of Theorem 1).

It is proved in [5, Lemma 4.3] that the set $Q$ is in one-to-one correspondence with the set of closed subsets of $S^{n-1}$ :

Lemma 3.2. [5,20] If $F$ is a closed non-empty subset of $S^{n-1}$,

$$
V_{F}(x)=\sup _{\lambda \in F} x \cdot \lambda
$$

belongs to $Q$; the map $F \mapsto V_{F}$ is one-to-one, and its inverse is the map

$$
w \in Q \mapsto F=\left\{x \in S^{n-1} \subset \mathbb{R}^{n} \mid w(x)=1\right\} .
$$

In particular, the blow down of a convex solution $u$ of (1.1) is determined by the set of its lightlike directions

$$
L_{u}=\left\{x \in S^{n-1} \subset \mathbb{R}^{n} \mid V_{u}(x)=1\right\} .
$$

As in [5], let us identify the unit ball $B_{1}(0) \subset \mathbb{R}^{n}$ with the Klein model of the hyperbolic geometry $\left\{(x, 1) \in \mathbb{L}^{n+1},|x|<1\right\}$; the Gauß map of the graph of an entire spacelike function $u$ in the natural chart $x \mapsto(x, u(x))$ is then simply the function $\mathbb{R}^{n} \rightarrow B_{1}(0), x \mapsto D u(x)$ (see [5, Lemma 4.5]). We also identify $S^{n-1}$ with the ideal boundary at infinity of the Klein model $B_{1}(0)$. The following lemma holds:

Lemma 3.3. The image of the Gauß map of the graph of an admissible solution $u$ of (1.1) is the convex hull in $B_{1}(0)$ of the set $L_{u}$. 
Proof. The proofs of Lemma 4.4 and Lemma 4.6 of [20] given there for the prescribed constant mean curvature equation extend to the prescribed constant Gauß curvature equation without modification. See also [15].

\section{The Construction of the Barriers}

In this section we describe known examples of entire hypersurfaces of constant Gauß curvature, the semitroughs, constructed for $n=2$ by Jun-ichi Hano and Katsumi Nomizu in [16] and for $n \geq 3$ by Bo Guan, Huai-Yu Jian and Richard Schoen in [15]. We prove some of their properties that we finally use to construct the barriers.

4.1. The Semitroughs. Let us first recall the properties of the standard semitrough constructed in [16] $(n=2)$ and in [15] $(n \geq 3)$ (see also [5] for the construction of the semitroughs with constant mean curvature): it is the graph $M$ of a function $u$ of the form

$$
u\left(x^{1}, x^{2}, \ldots, x^{n}\right)=\sqrt{f\left(x^{1}\right)^{2}+\left|x^{\prime}\right|^{2}},
$$

where $x^{\prime}=\left(x^{2}, \ldots, x^{n}\right)$, and where $f$ satisfies the following prescribed Gauß curvature equation:

$$
\frac{f^{\prime \prime}}{f^{n-1}\left(1-f^{\prime 2}\right)^{\frac{n+2}{2}}}=1,
$$

i.e., graph $u$ has Gauß curvature equal to one. Integrating equation (4.2), we get

$$
\left(1-f^{\prime 2}\right)^{-\frac{n}{2}}-f^{n} \equiv c
$$

where $c=\left(1-f^{\prime}(0)^{2}\right)^{-\frac{n}{2}}-f(0)^{n}$. Choosing $a \in(0, \infty)$ and $b \in(0,1)$ such that $\left(1-b^{2}\right)^{-\frac{n}{2}}-a^{n}=1$, it is proved in [15] that equation (4.3) has a unique solution $f: \mathbb{R} \rightarrow \mathbb{R}$ such that $f(0)=a, f^{\prime}(0)=b$. The function $f$ has the following properties (see [15, Section 2]):

(i) $f>0,1>f^{\prime}>0, f^{\prime \prime}>0$;

(ii) $\lim _{t \rightarrow-\infty} f(t)=0, \lim _{t \rightarrow-\infty} f^{\prime}(t)=0$;

(iii) $\lim _{t \rightarrow+\infty} \sqrt{1+t^{2}}-f(t)=\lambda<0$.

Considering $f(t+\lambda)$ instead of $f(t)$, we may suppose instead of (iii) that

(iii') $\lim _{t \rightarrow+\infty} \sqrt{1+t^{2}}-f(t)=0$. 
We thus get an entire function $u$ given by (4.1) whose graph has constant Gauß curvature 1, such that

$$
D u\left(\mathbb{R}^{n}\right)=\left\{x \in B_{1}(0): x^{1}>0\right\},
$$

and

$$
\lim _{|x| \rightarrow \infty} u(x)-V_{\overline{B^{+}}}(x)=0,
$$

where $\overline{B^{+}}$is the closed ball in $S^{n-1}$ defined by $\overline{B^{+}}=\left\{x \in S^{n-1}: x^{1} \geq 0\right\}$.

Definition 4.1. The standard semitrough is the entire convex spacelike function $u$ whose graph has constant Gauß curvature 1 and is asymptotic to $V_{\overline{B^{+}}}$, where

$$
\overline{B^{+}}=\left\{x \in S^{n-1}: x^{1} \geq 0\right\} .
$$

By the comparison principle, Lemma A.1, such a function is unique. It is given by (4.1), where $f$ solves (4.2) and satisfies (i), (ii), and (iii'). In the Klein model, the image of the Gauß map of its graph is $B_{1}(0) \cap\left\{x^{1}>0\right\}$.

Let us denote by $d_{S}$ the natural distance on $S^{n-1}$ : for $x, y \in S^{n-1}$, we have

$$
d_{S}(x, y)=\arccos (x \cdot y) \in[0, \pi]
$$

where the dot stands for the canonical scalar product in $\mathbb{R}^{n}$. A ball in $S^{n-1}$ is a ball in the metric space $\left(S^{n-1}, d_{S}\right)$, i.e. a set

$$
B=\left\{x \in S^{n-1}: d_{S}\left(x, x_{0}\right)<\delta\right\},
$$

where $x_{0}$ is some point of $S^{n-1}$ (the center of the ball) and where $\delta$ is a positive constant; $\delta$ is the radius of $B$, also denoted by $\delta(B) . \bar{B}$ will denote the closure of $B$ in $S^{n-1}$, and $B^{c}$ the complement of $B$ in $S^{n-1}$. We note that $B^{c}$ is a ball of radius $\pi-\delta(B)$.

Applying Lorentz transformations and homotheties we thus get from the existence of $u$ the existence of the so-called semitroughs. (This is done implicitly for the mean curvature case in $[5,20]$.): for every ball $\bar{B}$ of $S^{n-1}$ there exists an entire function $z_{\bar{B}}$ on $\mathbb{R}^{n}$ whose graph is a hypersurface with constant Gauß curvature $k>0$ in $\mathbb{L}^{n+1}$ which is asymptotic to $V_{\bar{B}}$. The image of the Gauß map of such a hypersurface is the convex hull of $\bar{B}$ in $B_{1}(0)$. We also observe that, by the comparison principle (Lemma A.1), the entire convex hypersurface with given Gauß curvature $k$ asymptotic to $V_{\bar{B}}$ is unique.

The next lemma gathers the properties of the semitroughs that we will use to construct the barriers (see Section 4.2 below).

Lemma 4.2. Let $\bar{B}$ be a closed ball of $S^{n-1}$ such that $\pi-\delta_{0} \geq \delta(\bar{B}) \geq \delta_{0}$ for some $\delta_{0}>0$ and let $z_{\bar{B}}$ be the semitrough with Gauß curvature $k$ which is asymptotic to $V_{\bar{B}}$. The following holds: 
(i) Let $h_{k}(x)=\sqrt{k^{-\frac{2}{n}}+|x|^{2}}$. Then

$$
h_{k} \geq z_{\bar{B}}>V_{\bar{B}}
$$

and

$$
z_{\bar{B}}(x)-V_{\bar{B}}(x) \rightarrow 0 \text { as }|x| \rightarrow \infty
$$

uniformly in $\bar{B}$ such that $\pi-\delta_{0} \geq \delta(\bar{B}) \geq \delta_{0}$.

(ii) For all compact sets $K \subset \mathbb{R}^{n}$ there exists $\delta=\delta\left(K, \delta_{0}, k\right)>0$ such that: for all $x \in K$,

$$
z_{\bar{B}}(x) \geq V_{\bar{B}}(x)+\delta
$$

(iii) For all compact sets $K \subset \mathbb{R}^{n}$ there exists $\vartheta_{K}=\vartheta_{K}\left(K, \delta_{0}, k\right) \in(0,1]$ such that: for all $x, y \in K$,

$$
\left|z_{\bar{B}}(x)-z_{\bar{B}}(y)\right| \leq\left(1-\vartheta_{K}\right)|x-y| .
$$

(iv) For $i=1,2$, let $z_{i, \bar{B}}$ denote the semitroughs with Gauß curvature $k_{i}$ which are asymptotic to $V_{\bar{B}}$. If $k_{1}>k_{2}$, then $z_{2, \bar{B}}>z_{1, \bar{B}}$. More precisely, for all compact sets $K \subset \mathbb{R}^{n}$ there exists $\delta>0$ such that, for all $x \in K$,

$$
z_{2, \bar{B}}(x) \geq z_{1, \bar{B}}(x)+\delta
$$

The constant $\delta$ depends on $K, \delta_{0}, k_{1}, k_{2}$, and $k_{1}-k_{2}$.

Proof. We first prove (i). We may suppose that $k=1$. For the standard semitrough $u$ (c.f. Definition 4.1 above) we have

$$
h_{1} \geq u>V_{\overline{B^{+}}} \text {. }
$$

Let $\psi$ be a Lorentz transformation which maps $\overline{B^{+}}$to $\bar{B}$ (recall that the Lorentz transformations act as the conformal maps on the boundary $S^{n-1}$ of the Klein model, which is identified with the projective lightcone in $\mathbb{L}^{n+1}$ ). The function $\psi$ maps the graphs of $V_{\overline{B^{+}}}, u$ and $h_{1}$ to the graphs of $V_{\bar{B}}, z_{\bar{B}}$ and $h_{1}$ respectively. This implies the inequalities in (4.5).

We then focus on the study of the limit. Let us first choose coordinates such that the ball $\bar{B}$ is centered around $(1,0, \ldots, 0)$ and such that the semitrough $z_{\bar{B}}$ is the image of the standard semitrough $u$ under the Lorentz transformation

$$
\left(\begin{array}{c}
x^{\prime 1} \\
x^{\prime n+1}
\end{array}\right)=\left(\begin{array}{cc}
\cosh (\varphi) & \sinh (\varphi) \\
\sinh (\varphi) & \cosh (\varphi)
\end{array}\right)\left(\begin{array}{c}
x^{1} \\
x^{n+1}
\end{array}\right), x^{\prime i}=x^{i} \text { for } 2 \leq i \leq n
$$

The radius $\delta(\bar{B})$ of $\bar{B}$ is given by $\delta(\bar{B})=\arccos (\tanh (\varphi))$, and the condition $\pi-\delta_{0} \geq \delta(\bar{B}) \geq \delta_{0}$ reads

$$
-\varphi_{0} \leq \varphi \leq \varphi_{0}
$$

where $\varphi_{0}=\tanh ^{-1}\left(\cos \left(\delta_{0}\right)\right)$ is a finite number. Now the rest of the claim follows from Lemma D.1. 
For the proof of (ii)-(iv), we observe that the properties (4.7), (4.8) and (4.9) clearly hold on the compact set $K$, for each closed ball $\bar{B}$. Keeping the notations from above, they are uniform in $\varphi \in\left[-\varphi_{0}, \varphi_{0}\right]$ by continuity and compactness $\left(\varphi_{0}\right.$ is finite).

4.2. The Barriers. The aim of this section is to construct two barriers for the prescribed Gauß curvature equation with given asymptotics. We obtain the following existence theorem.

Theorem 4.3. Let $S^{n-1}$ denote the ideal boundary at infinity of hyperbolic space and $F \subset S^{n-1}$ a non-empty closed subset. Assume that $F$ is of the following form: if $n \geq 3, F$ is the closure of some open subset of $S^{n-1}$ with $C^{1,1}$-boundary; if $n=2, F$ is a finite union of non-trivial intervals on the unit circle. Define $V_{F}: \mathbb{R}^{n} \rightarrow \mathbb{R}$ by

$$
V_{F}(x):=\sup _{\lambda \in F} x \cdot \lambda .
$$

Let $k_{1}>k_{2}$ be two positive constants. Then there exist two functions $\underline{u}$, $\bar{u}: \mathbb{R}^{n} \rightarrow \mathbb{R}$, such that $\underline{u}$ is a convex subsolution to the equation of prescribed Gauß curvature $k_{1}$ and $\bar{u}$ is a supersolution to the equation of prescribed Gauß curvature $k_{2}$. More precisely, $\underline{u}$ is the supremum of functions $u$ : $\mathbb{R}^{n} \rightarrow \mathbb{R}$ which are strictly convex, strictly spacelike, and fulfill the equation $K[u]=k_{1}$. Similarly, $\bar{u}$ is the infimum of strictly convex strictly spacelike functions $u$ such that $K[u]=k_{2}$. The barriers $\underline{u} \leq \bar{u}$ have the following properties:

$$
\left|\underline{u}(x)-V_{F}(x)\right|+\left|\bar{u}(x)-V_{F}(x)\right| \rightarrow 0 \quad \text { as }|x| \rightarrow \infty .
$$

(ii) For every compact subset $K \subset \mathbb{R}^{n}$, there exists a constant $\vartheta>0$ such that for every $x, y \in K$

$$
\begin{aligned}
& |\underline{u}(x)-\underline{u}(y)| \leq(1-\vartheta)|x-y|, \\
& |\bar{u}(x)-\bar{u}(y)| \leq(1-\vartheta)|x-y| .
\end{aligned}
$$

(iii) For every compact subset $K \subset \mathbb{R}^{n}$, there exists a constant $\delta>0$ such that for every $x \in K$

$$
V_{F}(x)+\delta \leq \underline{u}(x) \leq \bar{u}(x)-\delta .
$$

By construction, $\underline{u}$ is the supremum of subsolutions of the form $z_{1, \bar{B}}$. So it is a subsolution in the viscosity sense. When we wish to compare a solution with $\underline{u}$, we can apply the comparison principle for the functions $z_{1, \bar{B}}$ and show that $u \geq z_{1, \bar{B}}$ for each $B$ considered. Then we obtain that $u \geq \underline{u}$. The situation for $\bar{u}$ is similar.

The following lemma states that the barriers obtained in Theorem 4.3 guarantee that our solutions $u$ have the desired asymptotics at infinity. 
Lemma 4.4. Let $F, \underline{u}$, and $\bar{u}$ be as in Theorem 4.3. Let $u: \mathbb{R}^{n} \rightarrow \mathbb{R}$ be a smooth convex function with $\underline{u} \leq u \leq \bar{u}$. Then the Gauß map image of the hypersurface graph $u$ is the hyperbolic space convex hull of $F$, i.e. the convex hull of $F$ in hyperbolic space.

Proof. Since $\underline{u} \leq u \leq \bar{u}$ with $\underline{u}, \bar{u}$ asymptotic to $V_{F}$, we have

$$
u(x)-V_{F}(x) \rightarrow 0 \text { as }|x| \rightarrow \infty .
$$

Thus $V_{u}=V_{F}$ and $F$ is the set of lightlike directions of $u$. Lemma 3.3 implies the result.

The end of this section is devoted to the proof of Theorem 4.3. We first state preliminary lemmas.

Under the hypotheses of Theorem 4.3, we obtain the following technical properties which essentially rely on the $C^{1,1}$ regularity of $F$ :

Lemma 4.5. Let $d_{S}$ be the natural distance on the sphere $S^{n-1}$. For $F$ as above, there exists $\delta_{0}>0$ such that the following holds:

(i) $F$ and $\overline{F^{c}}$ are the union of closed balls of $S^{n-1}$ of radius $\delta_{0}$;

(ii) For every $x \in \overline{F^{c}}$, there exists a closed ball $B$ with radius bounded below by $\delta_{0}$ which contains $x$ and is contained in $\overline{F^{c}}$ such that $d_{S}\left(x, B^{c}\right)=$ $d_{S}(x, F)$.

Proof. If $n=2$ these properties are evident and we focus on the case $n \geq 3$. Since the boundary of $F$ is compact and $C^{1,1}, F$ and $\overline{F^{c}}$ are a union of closed balls of radius $\delta_{0}$ for some $\delta_{0}>0$ sufficiently small. Moreover, we may choose $\delta_{0}$ smaller such that the following tubular neighborhood property holds: for every $x \in \overline{F^{c}}$, if $d_{S}(x, F) \leq \delta_{0}$ then there exists a unique $y \in F$ with $d_{S}(x, F)=d_{S}(x, y)$, and, denoting by $N$ the exterior normal of $F$ at $y$, $x$ may be written $x=\cos (\delta) y+\sin (\delta) N$ for some $\delta \in\left[0, \delta_{0}\right]$. We first assume that $x \in \overline{F^{c}}$ is such that $d_{S}(x, F) \leq \delta_{0}$ : if $B$ is the closed ball tangent to $F$ at $y$, of radius $\delta_{0}$ and exterior to $F$, then $B \subset \overline{F^{c}}$ and $d_{S}\left(x, B^{c}\right)=d_{S}(x, F)$. If now $x$ is such that $d_{S}(x, F) \geq \delta_{0}$, we take the closed ball of radius $d_{S}(x, F)$ and center $x$.

Let $k_{1}>k_{2}$ be two positive constants. We suppose that the set $F$ in $S^{n-1}$ satisfies the hypotheses of Theorem 4.3 and we fix $\delta_{0}>0$ as in Lemma 4.5. Let us define

$$
\underline{u}(x)=\sup _{\substack{\bar{B} \subset F \\ \delta(\bar{B}) \geq \delta_{0}}} z_{1, \bar{B}}(x) \text { and } \quad \bar{u}(x)=\inf _{\substack{\bar{B} \supset F \\ \delta(\bar{B}) \leq \pi-\delta_{0}}} z_{2, \bar{B}}(x),
$$

where $z_{1, \bar{B}}$ (resp. $z_{2, \bar{B}}$ ) is the semitrough asymptotic to $V_{\bar{B}}$ whose curvature is $k_{1}$ (resp. $k_{2}$ ). To study the properties of $\underline{u}$ and $\bar{u}$, we will need the following descriptions of $V_{F}$ : 
Lemma 4.6. For every closed subset $F$ of $S^{n-1}$ and for all $x \in S^{n-1}$,

$$
V_{F}(x)=\cos \left(d_{S}(x, F)\right),
$$

where $d_{S}$ denotes the natural distance on the sphere $S^{n-1}$.

In particular, if $F$ and $F^{\prime}$ are two closed subsets of $S^{n-1}$ and if $x \in S^{n-1}$ is such that $d_{S}(x, F)=d_{S}\left(x, F^{\prime}\right)$, then $V_{F}(x)=V_{F^{\prime}}(x)$. Thus if $y_{0} \in F$ is such that $d_{S}(x, F)=d_{S}\left(x, y_{0}\right)$, we have $V_{F}(x)=x \cdot y_{0}$.

Proof. Let $y_{0} \in F$ be such that $d_{S}(x, F)=d_{S}\left(x, y_{0}\right)$. In view of (4.4), to prove the lemma, we have to prove that $V_{F}(x)=x \cdot y_{0}$. By definition, $V_{F}(x)=\sup _{\lambda \in F} x \cdot \lambda$. Since $y_{0} \in F$, we have $V_{F}(x) \geq x \cdot y_{0}$. For every $\lambda \in F$, $d_{S}(x, \lambda) \geq d_{S}\left(x, y_{0}\right)$. Thus $x \cdot \lambda \leq x \cdot y_{0}$ and $V_{F}(x) \leq x \cdot y_{0}$.

Lemma 4.7. Under the hypotheses on $F$ made above, we have: for all $x \in \mathbb{R}^{n}$,

$$
V_{F}(x)=\inf _{\substack{\bar{B} \supset F \\ \delta(\bar{B}) \leq \pi-\delta_{0}}} V_{\bar{B}}(x)=\sup _{\substack{\bar{B} \subset F \\ \delta(\bar{B}) \geq \delta_{0}}} V_{\bar{B}}(x)
$$

Proof. We may suppose that $x \in S^{n-1}$. Since $V_{F_{1}} \leq V_{F_{2}}$ if $F_{1} \subset F_{2}$, we obviously have:

$$
\sup _{\substack{\bar{B} \subset F \\ \delta(\bar{B}) \geq \delta_{0}}} V_{\bar{B}}(x) \leq V_{F}(x) \leq \inf _{\substack{\bar{B} \supset F \\ \delta(\bar{B}) \leq \pi-\delta_{0}}} V_{\bar{B}}(x) .
$$

We first prove that $V_{F}(x) \geq \inf \left\{V_{\bar{B}}(x): \bar{B} \supset F, \delta(\bar{B}) \leq \pi-\delta_{0}\right\}:$ if $x \in F$, the result is obvious since $V_{F}(x)=V_{\bar{B}}(x)=1$ for every ball $\bar{B}$ containing $F$. We thus assume that $x \notin F$, and we consider a ball $B$ of $S^{n-1}$ with radius bounded below by $\delta_{0}$ which contains $x$ and is contained in $\overline{F^{c}}$, such that $d_{S}\left(x, B^{c}\right)=d_{S}(x, F)$ (Lemma 4.5). Thus, from Lemma 4.6, we have $V_{F}(x)=V_{\overline{B^{c}}}(x)$, and, since $F \subset \overline{B^{c}}$ with $\delta\left(\overline{B^{c}}\right) \leq \pi-\delta_{0}$, the desired inequality.

We now prove that $V_{F} \leq \sup \left\{V_{\bar{B}}(x): \bar{B} \subset F, \delta(\bar{B}) \geq \delta_{0}\right\}:$ if $x \in F, V_{F}(x)=$ 1. By the definition of $\delta_{0}$, there exists a ball $\bar{B} \subset F$ of radius $\delta_{0}$ which contains $x$. Since $V_{\bar{B}}(x)=1$ we obtain the result. If $x \notin F$, we consider $y_{0} \in F$ such that $d_{S}(x, F)=d_{S}\left(x, y_{0}\right)$ and a ball $\bar{B} \subset F$ of radius $\delta_{0}$ which contains $y_{0}$. We have $d_{S}(x, \bar{B})=d_{S}(x, F)$. Thus, from Lemma 4.6, we have $V_{F}(x)=V_{\bar{B}}(x)$, and thus the last inequality follows.

Proof of Theorem 4.3. For proving (ii), we observe that the inequalities (4.13) and (4.14) are direct consequences of Lemma 4.2 (iii), since the closed balls involved in the definition of $\underline{u}$ and $\bar{u}$ have radius between $\delta_{0}$ and $\pi-\delta_{0}$.

The second inequality in (4.15) is a direct consequence of Lemma 4.2 (iv), since $z_{2, \bar{B}} \leq z_{2, \overline{B^{\prime}}}$ if $\bar{B} \subset F \subset \overline{B^{\prime}}$ (to prove this last claim, note that $V_{\bar{B}} \leq V_{\overline{B^{\prime}}}$ 
and apply the comparison principle, Lemma A.1). The first inequality in (4.15) follows directly from Lemma 4.2 (ii) and Lemma 4.7.

We now prove (i). We first note that (4.15) implies that $V_{F}<\underline{u}<\bar{u}$ on $\mathbb{R}^{n}$. To prove (4.12), it is thus sufficient to prove that $\bar{u}(x)-V_{F}(x) \rightarrow 0$ as $|x| \rightarrow \infty$. If $\frac{x}{|x|} \in F$

$$
\bar{u}(x)-V_{F}(x)=\bar{u}(x)-|x| \leq h_{k_{2}}(x)-|x|
$$

where $h_{k_{2}}(x)=\sqrt{k_{2}^{-\frac{2}{n}}+|x|^{2}}$. Thus $\bar{u}(x)-V_{F}(x)$ tends to zero when $|x|$ tends to $\infty$, with $\frac{x}{|x|} \in F$. If $\frac{x}{|x|} \notin F$, we consider a ball $B$ in $S^{n-1}$ with radius bounded below by $\delta_{0}$, containing $\frac{x}{|x|}$ and contained in $F^{c}$ such that $d_{S}\left(x /|x|, B^{c}\right)=d_{S}(x /|x|, F)$, given by Lemma 4.5. From Lemma 4.6 we get $V_{F}(x)=V_{\overline{B^{c}}}(x)$. Since $F \subset \overline{B^{c}}$ and $\delta\left(\overline{B^{c}}\right) \leq \pi-\delta_{0}$, we thus obtain $0 \leq \bar{u}(x)-V_{F}(x) \leq z_{\overline{B^{c}}}(x)-V_{\overline{B^{c}}}(x)$. Since $\pi-\delta_{0} \geq \delta\left(\overline{B^{c}}\right) \geq \delta_{0}$, Lemma 4.2 (i) gives the result.

\section{LOCAL $C^{1}$-Estimates}

We have the following estimate which is independent of the differential equation.

Lemma 5.1. Let $\Omega \subset \mathbb{R}^{n}$ be a bounded open set. Let $u, \bar{u}, \psi: \Omega \rightarrow \mathbb{R}$ be strictly spacelike. Assume that near $\partial \Omega$, we have $\psi>\bar{u}$. Everywhere in $\Omega$ we assume that $u \leq \bar{u}$. Consider the set, where $u>\psi$. For every $x$ in that set, we get the following gradient estimate for $u$

$$
\frac{1}{\sqrt{1-|D u(x)|^{2}}} \leq \frac{1}{u(x)-\psi(x)} \cdot \sup _{\{u>\psi\}} \frac{\bar{u}-\psi}{\sqrt{1-|D \psi|^{2}}}
$$

This lemma allows to get uniform gradient estimates on the set, where $u-\psi$ is estimated from below by a positive constant. Note that this a priori estimate is extended in [3].

Proof of Lemma 5.1. Consider

$$
\frac{u-\psi}{\sqrt{1-|D u|^{2}}}
$$

in the set $\{u>\psi\}$. It vanishes at the boundary. Therefore it has an interior positive maximum. We deduce that also $\varphi$ defined by

$$
\varphi:=-\frac{1}{2} \log \left(1-|D u|^{2}\right)+\log (u-\psi)
$$

has an interior maximum. 
At that local maximum of $w$, we get

$$
0=\varphi_{i}=\frac{u^{k} u_{k i}}{1-|D u|^{2}}+\frac{u_{i}-\psi_{i}}{u-\psi}
$$

We deduce there that

$$
0=\varphi_{i} u^{i}=\frac{u^{k} u_{k i} u^{i}}{1-|D u|^{2}}+\frac{|D u|^{2}-\langle D \psi, D u\rangle}{u-\psi} .
$$

As $u$ is convex, we may drop the first term and obtain there

$$
|D u|^{2} \leq\langle D u, D \psi\rangle \leq|D u| \cdot|D \psi|
$$

It follows everywhere on $\{u>\psi\}$ that

$$
\frac{u-\psi}{\sqrt{1-|D u|^{2}}} \leq \sup _{\{u>\psi\}} \frac{u-\psi}{\sqrt{1-|D u|^{2}}} \leq \sup _{\{u>\psi\}} \frac{\bar{u}-\psi}{\sqrt{1-|D \psi|^{2}}} .
$$

We arrive at the estimate claimed above.

We now construct the function $\psi$. Let us fix $\lambda \in(0,1)$. We consider

$$
\underline{u}^{\lambda}(x):=\lambda \underline{u}\left(\frac{x}{\lambda}\right),
$$

where $\underline{u}$ is the lower barrier constructed in Section 4.2. Since $V_{F}$ is a homogeneous function of degree one, we have

$$
V_{F}<\underline{u}^{\lambda}<\underline{u} .
$$

Let $K \subset \mathbb{R}^{n}$ be a compact set, and $\delta>0$ a constant such that $\underline{u}-\underline{u}^{\lambda} \geq 2 \delta$ on $K$. We set $\psi=\underline{u}^{\lambda}+\delta$. We have $\psi \leq \underline{u}-\delta$ on $K$, and $\psi-\bar{u} \rightarrow \delta$ as $|x| \rightarrow \infty$, where $\bar{u}$ is the upper barrier constructed in Section 4.2 , since $\bar{u}$ is also asymptotic to $V_{F}$. The latter implies that $\psi>\bar{u}$ near the boundary of some bounded open set $\Omega \subset \mathbb{R}^{n}$ which contains $K$. Smoothing $\psi$ by a convolution, Lemma 5.1 gives the $C^{1}$ estimate on $K$ for every spacelike convex function $u$ between $\underline{u}$ and $\bar{u}$.

\section{LoCAl $C^{2}$-Estimates}

Similarly to [13, Chapter 17.7], we obtain local $C^{2}$-estimates.

Theorem 6.1. Let $\Omega \subset \mathbb{R}^{n}$ be a bounded domain, $l: \mathbb{R}^{n} \rightarrow \mathbb{R}$ be an affine linear function. Suppose that $u: \Omega \times[0, \infty)$ is strictly convex. Assume that $u$ is smooth, spacelike, $|u|+\frac{1}{1-|D u|^{2}}$ is uniformly bounded, and $l(x)-u(x, t)<0$ for $(x, t) \in \partial \Omega \times[0, \infty)$. If $\dot{u}$ is uniformly bounded and $u$ solves

$$
\dot{u}=\sqrt{1-|D u|^{2}}\left(\log \frac{\operatorname{det} D^{2} u}{\left(1-|D u|^{2}\right)^{\frac{n+2}{2}}}-\log f\right)
$$


in $\Omega \times[0, \infty)$, where $f$ is a positive constant, then

$$
(x, t) \mapsto \exp \left(\frac{\beta}{\sqrt{1-|D u|^{2}}}\right) \cdot \frac{l(x)-u(x, t)}{\sqrt{1-|D u|^{2}}} \cdot \max _{|\xi|=1} \frac{u_{i j} \xi^{i} \xi^{j}}{\left(\delta_{i j}-u_{i} u_{j}\right) \xi^{i} \xi^{j}},
$$

where indices of $u$ denote partial derivatives and $\beta$ is sufficiently large, is uniformly bounded in the set $\{(x, t) \in \Omega \times[0, \infty): l(x)>u(x, t)\}$ in terms of its sup at $t=0$ and the bounds assumed above. Invariantly, this quantity is rewritten as

$$
\left(l-\eta_{\alpha} X^{\alpha}\right) \cdot e^{\beta \tilde{v}} \cdot \max _{|\xi|=1} \frac{h_{i j} \xi^{i} \xi^{j}}{g_{i j} \xi^{i} \xi^{j}}
$$

where $l$ is extended trivially to $\mathbb{R}^{n+1}$ and $\eta_{\alpha}=(0, \ldots, 0,1)$.

Remark 6.2. If we consider solutions between barriers, we can control the set, where $l-u>1$. So Theorem 6.1 implies bounds on the principal curvatures and thus spatial $C^{2}$ - and $C^{2,1}$-bounds. If we consider a sequence of such functions, we thus get uniform $C^{2,1}$-bounds on $K \times[0, \infty)$ for compact sets $K$.

We can weaken the regularity assumption on our initial data $\left.u\right|_{t=0}$. If this function can be approximated in $C^{0}$ by $C^{2}$-functions (for which Theorem B.4 implies the existence of a solution) with bounded $\log K$, we can also obtain spatial $C^{2}$-bounds on the sequence as long as $t$ is uniformly bounded below by a positive constant. In order to prove this, one applies arguments as in the proof of Theorem 6.1 to the function

$$
\left(l-\eta_{\alpha} X^{\alpha}\right) \cdot e^{\beta \tilde{v}} \cdot\left(\max _{|\xi|=1} \frac{h_{i j} \xi^{i} \xi^{j}}{g_{i j} \xi^{i} \xi^{j}}\right)^{t} .
$$

Proof of Theorem 6.1. Assume that the function

$$
\left(l-\eta_{\alpha} X^{\alpha}\right) \cdot e^{\beta \tilde{v}} \cdot \max _{|\xi|=1} \frac{h_{i j} \xi^{i} \xi^{j}}{g_{i j} \xi^{i} \xi^{j}}
$$

attains a new positive maximum at some positive time and coordinates are chosen such that $\xi=e_{1}$ gives this maximal value there. Define

$$
w:=\log \left(l-\eta_{\alpha} X^{\alpha}\right)+\beta \tilde{v}+\log \frac{h_{11}}{g_{11}} .
$$

We will assume for the rest of the proof that $l-\eta_{\alpha} X^{\alpha}>0$ at the point considered.

Following [10], it suffices to apply the maximum principle to the function $w$ if we want to bound the expression above.

We have the evolution equation

$$
\frac{d}{d t}\left(l-\eta_{\alpha} X^{\alpha}\right)-F^{i j}\left(l-\eta_{\alpha} X^{\alpha}\right)_{; i j}=\left(l_{\alpha}-\eta_{\alpha}\right) \nu^{\alpha}(F-\hat{f}-n) .
$$


Combining (2.1), (2.2), and (2.4), we get

$$
\frac{d}{d t} h_{i j}-F^{k l} h_{i j ; k l}=-H h_{i j}+(F-\hat{f}+n) h_{i}^{k} h_{k j}-\tilde{h}^{k r} \tilde{h}^{l s} h_{k l ; i} h_{r s ; j} .
$$

In the maximum considered, we get

$$
\begin{aligned}
& 0 \leq \frac{d}{d t} w=\frac{l_{\alpha} \dot{X}^{\alpha}-\eta_{\alpha} \dot{X}^{\alpha}}{l-\eta_{\alpha} X^{\alpha}}+\beta \dot{\tilde{v}}+\frac{1}{h_{11}} \dot{h}_{11}-\frac{1}{g_{11}} \dot{g}_{11}, \\
& 0= w_{; i}=\frac{l_{\alpha} X_{; i}^{\alpha}-\eta_{\alpha} X_{; i}^{\alpha}}{l-\eta_{\alpha} X^{\alpha}}+\beta \tilde{v}_{; i}+\frac{1}{h_{11}} h_{11 ; i}, \\
& 0 \geq w_{; i j}=\frac{l_{\alpha} X_{; i j}^{\alpha}-\eta_{\alpha} X_{; i j}^{\alpha}}{l-\eta_{\alpha} X^{\alpha}}+\beta \tilde{v}_{; i j}+\frac{1}{h_{11}} h_{11 ; i j}-\frac{1}{h_{11}^{2}} h_{11 ; i} h_{11 ; j} \\
& \\
& \quad-\frac{\left(l_{\alpha} X_{; i}^{\alpha}-\eta_{\alpha} X_{; i}^{\alpha}\right)\left(l_{\beta} X_{; j}^{\beta}-\eta_{\beta} X_{; j}^{\beta}\right)}{\left(l-\eta_{\alpha} X^{\alpha}\right)^{2}}, \\
& 0 \leq \frac{1}{l-\eta_{\alpha} X^{\alpha}}\left(\frac{d}{d t}\left(l-\eta_{\alpha} X^{\alpha}\right)-F^{i j}\left(l-\eta_{\alpha} X^{\alpha}\right)_{; i j}\right)+\beta\left(\frac{d}{d t} \tilde{v}-F^{i j} \tilde{v}_{; i j}\right) \\
&+\frac{1}{h_{11}}\left(\frac{d}{d t} h_{11}-F^{i j} h_{11 ; i j}\right)+\frac{1}{h_{11}^{2}} F^{i j} h_{11 ; i} h_{11 ; j} \\
&+F^{i j} \frac{\left(l_{\alpha} X_{; i}^{\alpha}-\eta_{\alpha} X_{; i}^{\alpha}\right)\left(l_{\beta} X_{; j}^{\beta}-\eta_{\beta} X_{; j}^{\beta}\right)}{\left(l-\eta_{\alpha} X^{\alpha}\right)^{2}}-\frac{1}{g_{11}} \dot{g}_{11 .} .
\end{aligned}
$$

Let us use $C^{1}$-bounds and normal velocity bounds. Let us also assume that $h_{11} \gg 1$ at an interior maximum and $\beta \gg 1$. Then we conclude that

$$
\begin{aligned}
0 \leq & \frac{c}{l-\eta_{\alpha} X^{\alpha}}-\frac{\beta}{2} \tilde{v} H \\
& -\frac{1}{h_{11}} \tilde{h}^{k r} \tilde{h}^{l s} h_{k l ; 1} h_{r s ; 1}+\frac{1}{h_{11}^{2}} \tilde{h}^{i j} h_{11 ; i} h_{11 ; j} \\
& +\tilde{h}^{i j} \frac{\left(l_{\alpha} X_{; i}^{\alpha}-\eta_{\alpha} X_{; i}^{\alpha}\right)\left(l_{\beta} X_{; j}^{\beta}-\eta_{\beta} X_{; j}^{\beta}\right)}{\left(l-\eta_{\alpha} X^{\alpha}\right)^{2}} .
\end{aligned}
$$

We have

$$
\begin{aligned}
\tilde{v} & =\eta_{\alpha} \nu^{\alpha}, \\
\tilde{v}_{; i} & =\eta_{\alpha} \nu_{; i}^{\alpha}=\eta_{\alpha} h_{i}^{k} X_{; k}^{\alpha} .
\end{aligned}
$$

We may assume that we have chosen coordinates such that $h_{i j}$ is diagonal and $g_{i j}=\delta_{i j}$. We now want to consider the term that requires the most complicated estimates. Using the extremal condition

$$
0=\frac{l_{\alpha} X_{; i}^{\alpha}-\eta_{\alpha} X_{; i}^{\alpha}}{l-\eta_{\alpha} X^{\alpha}}+\beta \tilde{v}_{; i}+\frac{1}{h_{11}} h_{11 ; i}
$$


we get

$$
\begin{aligned}
& \tilde{h}^{i j} \frac{\left(l_{\alpha} X_{; i}^{\alpha}-\eta_{\alpha} X_{; i}^{\alpha}\right)\left(l_{\beta} X_{; j}^{\beta}-\eta_{\beta} X_{; j}^{\beta}\right)}{\left(l-\eta_{\alpha} X^{\alpha}\right)^{2}} \\
& =\frac{\left(l_{\alpha} X_{; 1}^{\alpha}-\eta_{\alpha} X_{; 1}^{\alpha}\right)\left(l_{\beta} X_{; 1}^{\beta}-\eta_{\beta} X_{; 1}^{\beta}\right)}{h_{11}\left(l-\eta_{\alpha} X^{\alpha}\right)^{2}} \\
& +\sum_{i>1} \tilde{h}^{i i} \frac{\left(l_{\alpha} X_{; i}^{\alpha}-\eta_{\alpha} X_{; i}^{\alpha}\right)\left(l_{\beta} X_{; i}^{\beta}-\eta_{\beta} X_{; i}^{\beta}\right)}{\left(l-\eta_{\alpha} X^{\alpha}\right)^{2}} \\
& \leq \frac{c}{h_{11}\left(l-\eta_{\alpha} X^{\alpha}\right)^{2}}+\sum_{i>1} \tilde{h}^{i i}\left(\beta \tilde{v}_{; i}+\frac{1}{h_{11}} h_{11 ; i}\right)^{2} \\
& =\frac{c}{h_{11}\left(l-\eta_{\alpha} X^{\alpha}\right)^{2}}+\sum_{i>1} \tilde{h}^{i i} \beta^{2} \tilde{v}_{; i}^{2}+2 \sum_{i>1} \tilde{h}^{i i} \beta \tilde{v}_{; i} \frac{1}{h_{11}} h_{11 ; i} \\
& +\sum_{i>1} \frac{1}{h_{11}^{2}} \tilde{h}^{i i} h_{11 ; i} h_{11 ; i} \\
& =\frac{c}{h_{11}\left(l-\eta_{\alpha} X^{\alpha}\right)^{2}}+\sum_{i>1} \tilde{h}^{i i} \beta^{2} \tilde{v}_{; i}^{2}-2 \sum_{i>1} \tilde{h}^{i i} \beta^{2} \tilde{v}_{; i}^{2} \\
& -2 \sum_{i>1} \tilde{h}^{i i} \beta \tilde{v}_{; i} \frac{l_{\alpha} X_{; i}^{\alpha}-\eta_{\alpha} X_{; i}^{\alpha}}{l-\eta_{\alpha} X^{\alpha}}+\sum_{i>1} \frac{1}{h_{11}^{2}} \tilde{h}^{i i} h_{11 ; i} h_{11 ; i} \\
& \leq \frac{c}{h_{11}\left(l-\eta_{\alpha} X^{\alpha}\right)^{2}} \\
& -2 \sum_{i>1} \tilde{h}^{i i} \beta \tilde{v}_{; i} \frac{l_{\alpha} X_{; i}^{\alpha}-\eta_{\alpha} X_{; i}^{\alpha}}{l-\eta_{\alpha} X^{\alpha}}+\sum_{i>1} \frac{1}{h_{11}^{2}} \tilde{h}^{i i} h_{11 ; i} h_{11 ; i} .
\end{aligned}
$$

We get

$$
\begin{aligned}
& -\frac{2 \beta}{l-\eta_{\alpha} X^{\alpha}} \sum_{i>1} \tilde{h}^{i i} \tilde{v}_{; i}\left(l_{\alpha} X_{; i}^{\alpha}-\eta_{\alpha} X_{; i}^{\alpha}\right) \\
= & -\frac{2 \beta}{l-\eta_{\alpha} X^{\alpha}} \sum_{i>1} \tilde{h}^{i i} h_{i i} \eta_{\alpha} X_{; i}^{\alpha}\left(l_{\beta} X_{; i}^{\beta}-\eta_{\beta} X_{; i}^{\beta}\right) \\
= & -\frac{2 \beta}{l-\eta_{\alpha} X^{\alpha}} \sum_{i>1}\left(l_{\beta} X_{; i}^{\beta}-\eta_{\beta} X_{; i}^{\beta}\right) X_{; i}^{\alpha} \eta_{\alpha} \\
\leq & \frac{2 \beta c}{l-\eta_{\alpha} X^{\alpha}} .
\end{aligned}
$$


So we obtain

$$
\begin{aligned}
\tilde{h}^{i j} \frac{\left(l_{\alpha} X_{; i}^{\alpha}-\eta_{\alpha} X_{; i}^{\alpha}\right)\left(l_{\beta} X_{; j}^{\beta}-\eta_{\beta} X_{; j}^{\beta}\right)}{\left(l-\eta_{\alpha} X^{\alpha}\right)^{2}} \leq & \frac{c}{h_{11}\left(l-\eta_{\alpha} X^{\alpha}\right)^{2}}+\frac{2 \beta c}{l-\eta_{\alpha} X^{\alpha}} \\
& +\sum_{i>1} \frac{1}{h_{11}^{2}} \tilde{h}^{i i} h_{11 ; i} h_{11 ; i} .
\end{aligned}
$$

In a local maximum of $w$, we deduce that

$$
\begin{aligned}
0 \leq & \frac{c(\beta)}{l-\eta_{\alpha} X^{\alpha}}-\frac{\beta}{2} \tilde{v} H \\
& -\frac{1}{h_{11}} \tilde{h}^{k r} \tilde{h}^{l s} h_{k l ; 1} h_{r s ; 1}+\frac{1}{h_{11}^{2}} \tilde{h}^{i j} h_{11 ; i} h_{11 ; j} \\
& +\frac{c}{h_{11}\left(l-\eta_{\alpha} X^{\alpha}\right)^{2}}+\sum_{i>1} \frac{1}{h_{11}^{2}} \tilde{h}^{i i} h_{11 ; i} h_{11 ; i} .
\end{aligned}
$$

According to the Codazzi equations, $h_{i j ; k}$ is symmetric in all indices. Thus

$$
\begin{aligned}
& -\frac{1}{h_{11}} \sum_{i, j} \tilde{h}^{i i} \tilde{h}^{j j} h_{i j ; 1}^{2}+\frac{1}{h_{11}^{2}} \sum_{i} \tilde{h}^{i i} h_{11 ; i}^{2}+\sum_{i>1} \frac{1}{h_{11}^{2}} \tilde{h}^{i i} h_{11 ; i}^{2} \\
= & -\frac{1}{h_{11}} \sum_{r, s>1} \tilde{h}^{r r} \tilde{h}^{s s} h_{r s ; 1}^{2} \leq 0 .
\end{aligned}
$$

Therefore we get

$$
0 \leq \frac{c(\beta)}{l-\eta_{\alpha} X^{\alpha}}-\frac{\beta}{2} \tilde{v} H+\frac{c}{h_{11}\left(l-\eta_{\alpha} X^{\alpha}\right)^{2}} .
$$

We use $\tilde{v} \geq 1$ and $h_{11} \leq H$. For fixed $\beta \gg 1$, we get

$$
h_{11}\left(l-\eta_{\alpha} X^{\alpha}\right) \leq c(\beta)+\frac{c}{h_{11}\left(l-\eta_{\alpha} X^{\alpha}\right)} .
$$

So we obtain an interior $C^{2}$-bound.

\section{Existence of Entire Solutions and Convergence}

Consider a sequence of functions $u_{R}$ as in Theorem B.4 solving

$$
\dot{u}_{R}=\sqrt{1-\left|D u_{R}\right|^{2}}\left(\log \frac{\operatorname{det} D^{2} u_{R}}{\left(1-\left|D u_{R}\right|^{2}\right)^{\frac{n+2}{2}}}-\log f\right)
$$

in $B_{R} \times[0, \infty)$. (In order to apply Theorem B.4, we may shift the barriers so that $\underline{u}<u_{0}<\bar{u}$.) Our uniform bounds on the normal velocity of graph $u_{R}$, the local spatial bounds in $C^{2}$ and higher order derivative estimates (due to Krylov, Safonov, and Schauder for positive times) imply that a subsequence converges in $C^{\infty}\left(\mathbb{R}^{n} \times(0, \infty)\right) \cap C^{1, \alpha ; 0, \alpha / 2}\left(\mathbb{R}^{n} \times[0, \infty)\right)$ for every $0<\alpha<1$ 
to a solution $u \in C^{\infty}\left(\mathbb{R}^{n} \times(0, \infty)\right) \cap C^{1,1 ; 0,1}\left(\mathbb{R}^{n} \times[0, \infty)\right)$ of the initial value problem

$$
\begin{cases}\dot{u}=\sqrt{1-|D u|^{2}}\left(\log \frac{\operatorname{det} D^{2} u}{\left(1-|D u|^{2}\right)^{\frac{n+2}{2}}}-\log f_{0}\right) & \text { in } \mathbb{R}^{n} \times(0, \infty), \\ u(\cdot, 0)=u_{0} & \text { in } \mathbb{R}^{n} .\end{cases}
$$

According to (B.4), the normal velocity $F-\hat{f}$ converges exponentially to zero. Therefore $u(\cdot, t)$ converges exponentially fast to a smooth strictly convex, strictly spacelike solution $\tilde{u}: \mathbb{R}^{n} \rightarrow \mathbb{R}$ of

$$
\frac{\operatorname{det} D^{2} \tilde{u}}{\left(1-|D \tilde{u}|^{2}\right)^{\frac{n+2}{2}}}=f_{0} \quad \text { in } \mathbb{R}^{n}
$$

with $\underline{u} \leq \tilde{u} \leq \bar{u}$. As $\bar{u}-\underline{u}$ converges to zero at infinity, the maximum principle, Lemma A.1, implies that $\tilde{u}$ is the only solution like that. This finishes the proof of Theorem 1.3.

Similarly, for proving Theorem 1.2, we first apply Theorem B.1 to construct a sequence of smooth strictly convex strictly spacelike functions $\varphi_{R}$ such that $\underline{u} \leq \varphi_{R} \leq \bar{u}$ and a sequence $u_{R}$ of solutions to (B.1) with $\Omega=B_{R}(0)$. The functions $\varphi_{R}$ can be obtained from mollifications of $\underline{u}$. Applying KrylovSafonov estimates and Schauder theory, we get higher derivative estimates. Therefore, we find a subsequence of $u_{R}$ that converges in $C^{\infty}\left(\mathbb{R}^{n}\right)$ to the solution $u$ of (1.1) with $\underline{u} \leq u \leq \bar{u}$. Thus we have proved Theorem 1.2.

\section{Appendix A. Comparison Principles}

We state a comparison principle for the Gauß curvature operator on spacelike functions defined on $\mathbb{R}^{n}$.

Lemma A.1. Let $u$ and $v$ be two strictly spacelike functions belonging to $C^{2}\left(\mathbb{R}^{n}\right)$. Let us assume that $v$ is strictly convex, the Gauß curvatures satisfy $K[u] \leq K[v]$ on $\mathbb{R}^{n}$ and $\liminf _{|x| \rightarrow \infty} u(x)-v(x) \geq 0$. Then $u \geq v$ on $\mathbb{R}^{n}$.

We omit the proof, which is very close to the proof of the following comparison principle for the parabolic operator

$$
P[u]=-\frac{\dot{u}}{\sqrt{1-|D u|^{2}}}+\log K[u]
$$

acting on spacelike functions defined on $\mathbb{R}^{n} \times(0, \infty)$.

Lemma A.2. Let $u$ and $v$ be two strictly spacelike functions belonging to $C^{2,1}\left(\mathbb{R}^{n} \times(0, \infty)\right) \cap C^{0}\left(\mathbb{R}^{n} \times[0, \infty)\right)$. Let us assume that $v(., t)$ is strictly convex for all $t$, that $P[u] \leq P[v]$ on $\mathbb{R}^{n} \times(0, \infty), u(., 0) \geq v(., 0)$ on $\mathbb{R}^{n}$, 
and $\liminf _{|x| \rightarrow \infty} \inf _{t \in\left[0, t_{0}\right]} u(x, t)-v(x, t) \geq 0$ for every $t_{0}>0$. Then $u \geq v$ on $\mathbb{R}^{n} \times[0, \infty)$

Proof. If there exists $\left(x_{0}, t_{0}\right) \in \mathbb{R}^{n} \times(0, \infty)$ such that $u\left(x_{0}, t_{0}\right)<v\left(x_{0}, t_{0}\right)$, let $\delta>0$ be such that $\delta<v\left(x_{0}, t_{0}\right)-u\left(x_{0}, t_{0}\right)$, and set $u_{\delta}=u+\delta$. Since $\liminf _{|x| \rightarrow \infty} \inf _{t \in\left[0, t_{0}\right]} u_{\delta}(x, t)-v(x, t) \geq \delta$ for all $t_{0}>0$, we see that $\Omega_{\delta, t_{0}}=$ $\left\{(x, t) \in \mathbb{R}^{n} \times\left(0, t_{0}\right) \mid u_{\delta}(x, t)<v(x, t)\right\}$ is a non-empty bounded open set. We have $v \leq u_{\delta}$ on the parabolic boundary of $\Omega_{\delta, t_{0}}$ and $P\left[u_{\delta}\right]=P[u] \leq P[v]$ in $\Omega_{\delta, t_{0}}$. Since $v$ is convex, the standard maximum principle, see also Remark B.3, implies that $v \leq u_{\delta}$ on $\Omega_{\delta, t_{0}}$, which is impossible.

\section{Appendix B. Existence of Solutions on Balls}

In order to prove existence of graphical solutions to the equation of prescribed Gauß curvature or to logarithmic Gauß curvature flow, we construct solutions $u_{R}$ with Dirichlet boundary conditions on balls $B_{R}(0)$ and let $R \rightarrow \infty$. Then we use local a priori estimates to show that a subsequence of the $u_{R}$ converges to an entire solution as $R \rightarrow \infty$. In this appendix, we describe how to obtain auxiliary solutions on balls.

B.1. Elliptic Dirichlet Problem. In the elliptic case, an existence theorem in Minkowski space is known [7] for convex domains. We will assume in Theorem B.1 and Theorem B.4 that the properties of being positive, convex, and spacelike are all strict and uniform. Assume also that the data are smooth with uniform a priori estimates.

Theorem B.1. Let $\Omega \subset \mathbb{R}^{n}$ be a bounded convex domain, $f: \Omega \rightarrow \mathbb{R}$ be positive, and $\varphi: \Omega \rightarrow \mathbb{R}$ be convex and spacelike. Then there exists a smooth convex spacelike solution $u: \Omega \rightarrow \mathbb{R}$ to the Dirichlet problem

$$
\begin{cases}\frac{\operatorname{det} D^{2} u}{\left(1-|D u|^{2}\right)^{\frac{n+2}{2}}}=f(x) & \text { in } \Omega, \\ u=\varphi & \text { on } \partial \Omega .\end{cases}
$$

Proof. See [7].

Remark B.2. Let us note that in the proof of Theorem B.1 in [7], Philippe Delanoë constructs spacelike convex barriers of given positive constant Gauß curvature that touch graph $\left.\varphi\right|_{\partial \Omega}$ from below at a given boundary point and lie below $\varphi$. We will use these barriers also in the parabolic setting. 
Remark B.3. If there exist upper and lower barriers, $\bar{u}$ and $\underline{u}$, which are strictly spacelike, strictly convex, of class $C^{2}$, and fulfill

$$
\begin{cases}\frac{\operatorname{det} D^{2} \bar{u}}{\left(1-|D \bar{u}|^{2}\right)^{\frac{n+2}{2}}} \leq f(x) \leq \frac{\operatorname{det} D^{2} \underline{u}}{\left(1-|D \underline{u}|^{2}\right)^{\frac{n+2}{2}}} & \text { in } \Omega \\ \bar{u} \geq \varphi \geq \underline{u} & \text { on } \partial \Omega\end{cases}
$$

then a solution $u$ to (B.1) fulfills $\underline{u} \leq u \leq \bar{u}$.

It even suffices to require that the differential inequality for $\bar{u}$ holds at those points, where $\bar{u}$ is spacelike and convex. This follows from the observation, see [12], that for a strictly convex strictly spacelike function, graph $u$ can touch graph $\bar{u}$ from below only in points, where $\bar{u}$ is strictly spacelike and strictly convex.

B.2. Parabolic Dirichlet Problem. We want to consider an initial value problem of the form

$$
\begin{cases}\dot{u}=\sqrt{1-|D u|^{2}}\left(\log \frac{\operatorname{det} D^{2} u}{\left(1-|D u|^{2}\right)^{\frac{n+2}{2}}}-\hat{f}_{0}\right) & \text { in } \Omega \times[0, \infty), \\ u=u_{0} & \text { on } \partial \Omega \times[0, \infty), \\ u=u_{0} & \text { on } \Omega \times\{0\} .\end{cases}
$$

In order to get a smooth solution, we have to modify $f_{0}$ such that compatibility conditions are fulfilled along $\partial \Omega \times\{0\}$. This is done in the following theorem. Note, however, that the evolution equation is unchanged outside of a neighborhood of $\partial \Omega$. Note further that $f_{0}$ is modified in such a way that the normal velocity $F-\hat{f}$ of the evolving hypersurfaces graph $u(\cdot, t)$ stays uniformly bounded along a sequence of balls $B_{R}=\Omega$ for which we consider such problems.

Theorem B.4. Let $\Omega:=B_{R}(0), R \geq 2$. Let $u_{0}: \Omega \rightarrow \mathbb{R}$ be convex and spacelike. Assume that $\underline{u}<u_{0}<\bar{u}$ with $\underline{u}$ and $\bar{u}$ as in Remark B.3. Extend $u_{0}$ by setting $u_{0}(x, t):=u_{0}(x)$. Let $f_{0}$ be a positive constant. Choose a smooth function $\eta: \bar{\Omega} \times \mathbb{R} \rightarrow[0,1]$ such that $\eta=0$ on $\left(B_{R-1}(0) \times \mathbb{R}\right) \cup \operatorname{graph} \underline{u} \cup$ graph $\bar{u}$ and $\eta=1$ near graph $\left.u_{0}\right|_{\partial \Omega}$. Choose also a smooth function $\zeta:[0, \infty) \rightarrow[0,1]$, independent of the other data, such that $\zeta(t)=1$ near $t=0$ and $\zeta(t)=0$ for $t \geq 1$. Define $f$ by

$\log f(x, u, t) \equiv \hat{f}:=\eta(x, u) \cdot \zeta\left(\frac{t}{\varepsilon}\right) \cdot\left(\log \frac{\operatorname{det} D^{2} u_{0}}{\left(1-\left|D u_{0}\right|^{2}\right)^{\frac{n+2}{2}}}-\log f_{0}\right)+\log f_{0}$.

If $\varepsilon>0$ is fixed sufficiently small, then there exists a uniformly strictly convex, uniformly strictly spacelike solution $u \in C^{\infty}(\bar{\Omega} \times[0, \infty))$ to the 
initial value problem

$$
\begin{cases}\dot{u}=\sqrt{1-|D u|^{2}}\left(\log \frac{\operatorname{det} D^{2} u}{\left(1-|D u|^{2}\right)^{\frac{n+2}{2}}}-\hat{f}(x, u, t)\right) & \text { in } \Omega \times[0, \infty), \\ u=u_{0} & \text { on } \partial \Omega \times[0, \infty), \\ u=u_{0} & \text { on } \Omega \times\{0\},\end{cases}
$$

such that the normal velocity $\frac{\dot{u}}{\sqrt{1-|D u|^{2}}}$ is uniformly bounded in terms of $f_{0}$ and $K\left[u_{0}\right]:=\frac{\operatorname{det} D^{2} u_{0}}{\left(1-\left|D u_{0}\right|^{2}\right)^{\frac{n+2}{2}}}$.

Proof. Short Time Existence: At the boundary, compatibility conditions of any order are fulfilled, so for a short time interval, we get a smooth solution. We will assume for the a priori estimates that a smooth solution exists for all positive times.

$C^{0}$-Estimates: The functions $\underline{u}$ and $\bar{u}$ are barriers and imply that $\underline{u} \leq u \leq$ $\bar{u}$. The $C^{0}$-bounds follow.

$C^{1}$-Estimates: As in [7], it suffices to prove gradient estimates at the boundary $\partial \Omega$. Note that the absolute value of the modified function $f$ is controlled independent of $R$. So we can use the lower barrier constructed in [7]. The maximal hypersurface found by Robert Bartnik and Leon Simon in [2] serves as an upper barrier for all boundary points simultaneously.

These barriers stay above or below the solution $u$ during the evolution. They are strictly spacelike and coincide at a given boundary point with the solution. Moreover, the tangential gradients of the barriers and of $u(\cdot, t)$ coincide at that boundary point, so we get $|D u| \leq 1-c$ everywhere along the boundary $\partial \Omega$ for some estimated positive constant $c$. Convexity implies interior $C^{1}$-bounds. Note that a positive lower bound on $c$ can be chosen so that it does not depend on $\varepsilon$.

Velocity Estimates: Under the evolution equation $\dot{X}=(F-\hat{f}(X, t)) \nu$, which is equivalent to the flow equation in (B.3), the normal velocity fulfills

$$
\frac{d}{d t}(F-\hat{f})-F^{i j}(F-\hat{f})_{; i j}=-(F-\hat{f}) H-\hat{f}_{\alpha} \nu^{\alpha}(F-\hat{f})-\hat{f}_{t} .
$$

Along the boundary, $F-\hat{f}$ vanishes. Define

$$
V(t):=\sup _{\operatorname{graph} u(\cdot, t)}|F-\hat{f}| .
$$

According to the maximum principle, we get (in the sense of difference quotients)

$$
\frac{d}{d t} V(t) \leq C \cdot V(t)+\frac{c_{1}}{\varepsilon},
$$


where $c_{1}$ depends only on $f_{0}$ and $K\left[u_{0}\right]$, but $C$ may grow in $R$ as $\mid u(x)-$ $\underline{u}(x)|+| u(x)-\bar{u}(x) \mid \rightarrow 0$ as $|x| \rightarrow \infty$. We may assume that $C \geq 1$. The function

$$
V(t)=\left(V(0)+\frac{c_{1}}{\varepsilon C}\right) e^{C t}-\frac{c_{1}}{\varepsilon C}
$$

solves this differential inequality with equality. If we pick $\varepsilon=\frac{1}{C}$, we get

$$
\sup _{0 \leq t \leq \varepsilon} V(t) \leq c\left(V(0), c_{1}\right)
$$

For the rest of the proof, we fix that value of $\varepsilon$. For $t \geq \varepsilon$, we get

$$
\frac{d}{d t}(F-\hat{f})-F^{i j}(F-\hat{f})_{; i j}=-(F-\hat{f}) H .
$$

As the evolving hypersurfaces are convex, we have $H \geq 0$ and the maximum principle implies

$$
V(t) \leq V(\varepsilon) \text { for all } t \geq \varepsilon
$$

This implies in particular a lower bound on $F$. Thus solutions stay convex. According to the geometric-arithmetic means inequality, there exists a positive lower bound on $H$, depending only on $c\left(V(0), c_{1}\right)$. We apply the maximum principle once again to the evolution equation for the normal velocity and get

$$
|F-\hat{f}| \leq c_{2} \cdot e^{-c_{3} \cdot t}
$$

with $c_{2}, c_{3}>0$ and $c_{2}+\frac{1}{c_{3}}$ bounded above in terms of $f_{0}$ and $K\left[u_{0}\right]$.

$C^{2}$-Estimates at the Boundary: The following $C^{2}$-estimates depend on $R$.

Tangential-Tangential Derivatives: These estimates follow directly from differentiating the boundary condition twice.

Tangential-Normal Derivatives: These a priori estimates follow from a standard barrier construction using

$$
\begin{aligned}
L w & =\dot{w}-v u^{i j} w_{i j}+\frac{1}{v}(F-\hat{f}) u^{i} w_{i}-\frac{n-2}{v} u^{i} w_{i}, \\
T u & =u_{r}+u_{n} \omega_{r}, \\
\vartheta & =d-\mu d^{2}, \\
\Theta & =A \vartheta+B\left|x-x_{0}\right|^{2} \pm T\left(u-u_{0}\right)+l,
\end{aligned}
$$

the differential inequality $L \Theta \geq 0$ in $\Omega_{\delta}=\Omega \cap B_{\delta}\left(x_{0}\right)$ and $\Theta \geq 0$ on $\partial \Omega_{\delta}$ with equality at $x_{0} \in \partial \Omega$. This bounds the normal derivatives of $T\left(u-u_{0}\right)$ and thus $u_{\tau \nu}$. Details can be found in [18] and many other papers.

Normal-Normal Derivatives: Sketch: This bound follows from techniques as in [21] and a similar barrier construction as above. 
Interior $C^{2}$-estimates: Consider the test function

$$
w:=\log H+\beta \tilde{v}
$$

for some $\beta \gg 1$. For a family of convex spacelike hypersurfaces moving with normal velocity $F-\hat{f}$, we get the following evolution equations

$$
\begin{aligned}
\frac{d}{d t} H-F^{i j} H_{; i j}= & n|A|^{2}-H^{2}-(F-\hat{f})|A|^{2}-H \hat{f}_{\alpha} \nu^{\alpha} \\
& -g^{i j} \tilde{h}^{k r} \tilde{h}^{l s} h_{k l ; i} h_{r s ; j}-\hat{f}_{\alpha \beta} X_{; i}^{\alpha} X_{; j}^{\beta} g^{i j}, \\
\frac{d}{d t} \tilde{v}-F^{i j} \tilde{v}_{; i j}= & -\tilde{v} H-\eta_{\alpha} X_{; i}^{\alpha} g^{i j} X_{; j}^{\beta} \hat{f}_{\beta}, \\
\frac{d}{d t} w-F^{i j} w_{; i j}= & \frac{1}{H}\left(\frac{d}{d t} H-F^{i j} H_{; i j}\right) \\
& +\frac{1}{H^{2}} F^{i j} H_{; i} H_{; j}+\beta\left(\frac{d}{d t} \tilde{v}-F^{i j} \tilde{v}_{; i j}\right) \\
= & (n-(F-\hat{f})) \frac{|A|^{2}}{H}-H-\hat{f}_{\alpha} \nu^{\alpha} \\
& -\frac{1}{H} \hat{f}_{\alpha \beta} X_{; i}^{\alpha} X_{; j}^{\beta} g^{i j}-\frac{1}{H} g^{i j} \tilde{h}^{k r} \tilde{h}^{l s} h_{k l ; i} h_{r s ; j} \\
& +\frac{1}{H^{2}} \tilde{h}^{i j} g^{k l} g^{r s} h_{k l ; i} h_{r s ; j}-\beta \tilde{v} H-\beta \eta_{\alpha} X_{; i}^{\alpha} g^{i j} X_{; j}^{\beta} \hat{f}_{\beta} .
\end{aligned}
$$

As the terms on the right-hand side involving derivatives of the second fundamental form are non-positive, we get in a point with $H \geq 1$ due to our $C^{1}$ a priori estimates

$$
\frac{d}{d t} w-F^{i j} w_{; i j} \leq c \cdot(1+H)-\beta(\tilde{v} H-c) .
$$

The maximum principle implies for $\beta \gg 1$ fixed sufficiently large a global bound on $H$. As $u$ is strictly convex, $u$ is bounded in $C^{2,1}$.

The estimates obtained so far guarantee that $u$ is uniformly strictly convex and spacelike.

Long Time Existence: The estimates of Krylov, Safonov, and Schauder imply bounds on higher derivatives of $u$. Thus a solution exists for all times (justifying our assumption above) and the theorem follows.

Remark B.5. There is a non-compact maximum principle by Klaus Ecker and Gerhard Huisken [9]. We can't apply this as the coefficients in our equation grow at infinity.

There is also a non-compact maximum principle that allows for coefficients growing at infinity [1]. We were not able to understand the proof. That's why we use the compact maximum principle for our auxiliary problems instead. 


\section{Appendix C. Velocity Bounds}

The estimates obtained here allow to consider initial data with $\log K\left[u_{0}\right]$ uniformly bounded below.

Theorem C.1. Let $\Omega \subset \mathbb{R}^{2}$ be a bounded domain, $\hat{f} \in \mathbb{R}$, and $l: \mathbb{R}^{2} \rightarrow \mathbb{R}$ be a spacelike affine linear function. Assume that $u \in C^{\infty}(\bar{\Omega} \times[0, \infty))$ is a solution of

$$
\dot{u}=\sqrt{1-|D u|^{2}}\left(\log \frac{\operatorname{det} D^{2} u}{\left(1-|D u|^{2}\right)^{\frac{n+2}{2}}}-\hat{f}\right) \text { in } \Omega \times[0, \infty),
$$

such that $l-u<0$ on $\partial \Omega \times[0, \infty)$ and $|u|+\frac{1}{1-|D u|^{2}}$ is uniformly bounded. Then $(l-u) \dot{u}$ is bounded above in the set $\{(x, t) \in \Omega \times[0, \infty): l(x)>u(x, t)\}$ in terms of an upper bound on $K[u(\cdot, 0)]$ on $\Omega$ and in terms of the bound assumed above.

Proof. We first suppose that the dimension is arbitrary. We will restrict it when necessary. Consider the test function

$$
w=\log \left(l-\eta_{\alpha} X^{\alpha}\right)+\log (F-\hat{f})+\log \tilde{v}
$$

where the notation is as above. We have

$$
\begin{aligned}
\frac{d}{d t} w-F^{i j} w_{; i j}= & \frac{1}{l-\eta_{\alpha} X^{\alpha}}\left(\frac{d}{d t}\left(l-\eta_{\alpha} X^{\alpha}\right)-F^{i j}\left(l-\eta_{\alpha} X^{\alpha}\right)_{; i j}\right) \\
& +\frac{1}{F-\hat{f}}\left(\frac{d}{d t} F-F^{i j} F_{; i j}\right)+\frac{1}{\tilde{v}}\left(\frac{d}{d t} \tilde{v}-F^{i j} \tilde{v}_{; i j}\right) \\
& +\frac{1}{\left(l-\eta_{\alpha} X^{\alpha}\right)^{2}} F^{i j}\left(l-\eta_{\alpha} X^{\alpha}\right)_{; i}\left(l-\eta_{\beta} X^{\beta}\right)_{; j} \\
& +\frac{1}{(F-\hat{f})^{2}} F^{i j} F_{; i} F_{; j}+\frac{1}{\tilde{v}^{2}} F^{i j} \tilde{v}_{; i} \tilde{v}_{; j} .
\end{aligned}
$$

At a point where $w$ attains a new maximum, we get

$$
0 \leq \frac{d}{d t} w-F^{i j} w_{; i j}
$$

and

$$
0=w_{; i}=\frac{\left(l-\eta_{\alpha} X^{\alpha}\right)_{; i}}{l-\eta_{\alpha} X^{\alpha}}+\frac{F_{; i}}{F-\hat{f}}+\frac{\tilde{v}_{; i}}{\tilde{v}}
$$


Using (C.1), (C.2), and (C.3), together with the evolution equations, see Section 2, we get

$$
\begin{aligned}
0 \leq & \frac{1}{l-\eta_{\alpha} X^{\alpha}}\left(l_{\alpha}-\eta_{\alpha}\right) \nu^{\alpha}(F-\hat{f}-n)-H-H \\
& +\frac{2}{\left(l-\eta_{\alpha} X^{\alpha}\right)^{2}} F^{i j}\left(l-\eta_{\alpha} X^{\alpha}\right)_{; i}\left(l-\eta_{\beta} X^{\beta}\right)_{; j} \\
& +\frac{2}{\tilde{v}^{2}} F^{i j} \tilde{v}_{; i} \tilde{v}_{; j}+\frac{2}{\left(l-\eta_{\alpha} X^{\alpha}\right) \tilde{v}} F^{i j}\left(l-\eta_{\alpha} X^{\alpha}\right)_{; i} \tilde{v}_{; j} .
\end{aligned}
$$

We may assume that $F-\hat{f} \geq n$ and $H \geq 1$ at the maximum of $w$. From the gradient estimate and $\tilde{v}_{; i}=\eta_{\alpha} h_{i}^{k} X_{; k}^{\alpha}$, we have

$$
\frac{2}{\left(l-\eta_{\alpha} X^{\alpha}\right) \tilde{v}} F^{i j}\left(l-\eta_{\alpha} X^{\alpha}\right)_{; i} \tilde{v}_{; j} \leq \frac{c}{l-\eta_{\alpha} X^{\alpha}} .
$$

The first term in (C.4) is non-positive: since $l$ and $u$ are spacelike,

$$
\left(l_{\alpha}-\eta_{\alpha}\right) \nu^{\alpha}=\frac{1}{\sqrt{1-|D u|^{2}}}\left(\sum_{i \leq n} l_{i} u_{i}-1\right) \leq 0 .
$$

Dropping this term in (C.4), using the inequality

$$
\frac{2}{\tilde{v}^{2}} F^{i j} \tilde{v}_{; i} \tilde{v}_{; j}-2 H \leq-\frac{2 H}{\tilde{v}^{2}},
$$

and (C.5), we thus get

$$
0 \leq-\frac{2 H}{\tilde{v}^{2}}+\frac{2}{\left(l-\eta_{\alpha} X^{\alpha}\right)^{2}} F^{i j}\left(l-\eta_{\alpha} X^{\alpha}\right)_{; i}\left(l-\eta_{\beta} X^{\beta}\right)_{; j}+\frac{c}{l-\eta_{\alpha} X^{\alpha}} .
$$

We thus obtain

$$
H \leq \frac{c_{2}}{\left(l-\eta_{\alpha} X^{\alpha}\right)^{2}} \sum_{i=1}^{n} \frac{1}{\lambda_{i}}+\frac{c_{1}}{l-\eta_{\alpha} X^{\alpha}} .
$$

Now we need to suppose that $n=2$. Multiplying by $\left(l-\eta_{\alpha} X^{\alpha}\right)^{2} K$ where $K$ is the Gauß curvature, we obtain

$$
\left(l-\eta_{\alpha} X^{\alpha}\right)^{2} K H \leq c_{2} H+c_{1}\left(l-\eta_{\alpha} X^{\alpha}\right) K .
$$

We first assume that

$$
\frac{1}{2}\left(l-\eta_{\alpha} X^{\alpha}\right)^{2} K H \geq c_{1}\left(l-\eta_{\alpha} X^{\alpha}\right) K .
$$

Inequality (C.6) then reads

$$
\left(l-\eta_{\alpha} X^{\alpha}\right)^{2} K \leq 2 c_{2} .
$$

Thus

$$
\left(l-\eta_{\alpha} X^{\alpha}\right)(\log K-\hat{f}) \leq\left(l-\eta_{\alpha} X^{\alpha}\right)\left(\log \left(2 c_{2}\right)-2 \log \left(l-\eta_{\alpha} X^{\alpha}\right)-\hat{f}\right) .
$$

The right hand side term is bounded above and so is $w$ at its maximum. 
We now suppose that (C.7) does not hold. This implies that

$$
\left(l-\eta_{\alpha} X^{\alpha}\right) H \leq 2 c_{1} .
$$

Applying the geometric-arithmetic means inequality $\sqrt{K} \leq \frac{1}{2} H$ gives an inequality as (C.8). We then conclude as above.

\section{Appendix D. Closeness at Infinity}

Lemma D.1. Let $u, v: \mathbb{R}^{n} \rightarrow \mathbb{R}$ be spacelike functions. For $\varphi \in \mathbb{R}$, let

$$
A^{\varphi}:=\left(\begin{array}{ccc}
\cosh \varphi & 0 & \sinh \varphi \\
0 & \text { Id } & 0 \\
\sinh \varphi & 0 & \cosh \varphi
\end{array}\right)
$$

be a Lorentz transformation. Then there exist spacelike function $u^{\varphi}, v^{\varphi}$ : $\mathbb{R}^{n} \rightarrow \mathbb{R}$ such that

$$
\operatorname{graph} u^{\varphi}=A^{\varphi}(\operatorname{graph} u) \text { and } \operatorname{graph} v^{\varphi}=A^{\varphi}(\operatorname{graph} v) \text {. }
$$

Let $\varphi_{0}>0$. If

$$
\lim _{|x| \rightarrow \infty} u(x)-v(x)=0
$$

then

$$
\lim _{|x| \rightarrow \infty} \sup _{|\varphi| \leq \varphi_{0}}\left|u^{\varphi}(x)-v^{\varphi}(x)\right|=0
$$

Proof. It is known that there exists a function $u^{\varphi}$ such that $A^{\varphi}($ graph $u)=$ graph $u^{\varphi}$ in the original coordinate system as a Lorentz transformation preserves the property of being spacelike. In this proof, we use Euclidean space, equipped with the standard norm. We claim first that the "Hausdorff distance at infinity" between graph $u^{\varphi}$ and graph $v^{\varphi}$ is zero:

$$
\lim _{R \rightarrow \infty} \sup _{\substack{a \in \operatorname{graph} u \varphi \backslash B_{R}(0) \\ b \in \operatorname{graph} v} \backslash B_{R}(0)} d\left(a, \operatorname{graph} v^{\varphi}\right)+d\left(b, \operatorname{graph} u^{\varphi}\right)=0 .
$$

Fix $\varepsilon>0$. Consider $R \gg 0$ and $a \in$ graph $u^{\varphi} \backslash B_{R}(0)$. Then there exists $\tilde{a} \in \operatorname{graph} u$ such that $A^{\varphi}(\tilde{a})=a$. We have $|\tilde{a}| \geq \frac{R}{c\left(\varphi_{0}\right)}$. As $u(x)-v(x) \rightarrow 0$ for $|x| \rightarrow \infty$, there exists $\tilde{b} \in \operatorname{graph} v$ such that $|\tilde{a}-\tilde{b}| \leq \varepsilon$ provided that $R$ is chosen sufficiently large. Define $b:=A^{\varphi}(\tilde{b}) \in \operatorname{graph} v^{\varphi}$. By the linearity of $A^{\varphi}$, there exists $L=L\left(\varphi_{0}\right)$ such that $\left|A^{\varphi}(p)-A^{\varphi}(q)\right| \leq L\left(\varphi_{0}\right) \cdot|p-q|$ for all $p, q \in \mathbb{R}^{n+1},|\varphi| \leq \varphi_{0}$. Thus $L \cdot \varepsilon \geq|a-b| \geq d\left(a, \operatorname{graph} v^{\varphi}\right)$.

Since $v^{\varphi}(x)-|y| \leq v^{\varphi}(x+y) \leq v^{\varphi}(x)+|y|$ for $y \in \mathbb{R}^{n}$, we see that for $x \in \mathbb{R}^{n}$ such that $\left|u^{\varphi}(x)-v^{\varphi}(x)\right| \geq \zeta>0$, we get $d\left(\left(x, u^{\varphi}(x)\right)\right.$, graph $\left.v^{\varphi}\right) \geq \frac{\zeta}{\sqrt{2}}$. Thus the lemma follows. 


\section{REFERENCES}

1. Mark A. S. Aarons, Mean curvature flow with a forcing term in Minkowski space, Calc. Var. Partial Differential Equations 25 (2006), no. 2, 205-246.

2. Robert Bartnik and Leon Simon, Spacelike hypersurfaces with prescribed boundary values and mean curvature, Commun. Math. Phys. 87 (1982), 131-152.

3. Pierre Bayard, Entire spacelike hypersurfaces of prescribed scalar curvature in Minkowski space, Calc. Var. Partial Differential Equations 26 (2006), no. 2, 245-264.

4. Albert Chau and Oliver C. Schnürer, Stability of gradient Kähler-Ricci solitons, Comm. Anal. Geom. 13 (2005), no. 4, 769-800.

5. Hyeong In Choi and Andrejs Treibergs, Gauss maps of spacelike constant mean curvature hypersurfaces of Minkowski space, J. Differential Geom. 32 (1990), no. 3, 775-817.

6. Julie Clutterbuck, Oliver C. Schnürer, and Felix Schulze, Stability of translating solutions to mean curvature flow, Calc. Var. Partial Differential Equations, to appear, arXiv:math.AP/0509372.

7. Philippe Delanoë, The Dirichlet problem for an equation of given Lorentz-Gaussian curvature, Ukrainian Math. J. 42 (1990), no. 12, 1538-1545, translation from Ukr. Mat. Zh. 42 (1990), no.12, 1704-1710.

8. Klaus Ecker, Interior estimates and longtime solutions for mean curvature flow of noncompact spacelike hypersurfaces in Minkowski space, J. Differ. Geom. 46 (1997), no. 3, 481-498.

9. Klaus Ecker and Gerhard Huisken, Interior estimates for hypersurfaces moving by mean curvature, Invent. Math. 105 (1991), no. 3, 547-569.

10. Claus Gerhardt, Closed Weingarten hypersurfaces in Riemannian manifolds, J. Differential Geom. 43 (1996), no. 3, 612-641.

11. Claus Gerhardt, Hypersurfaces of prescribed curvature in Lorentzian manifolds, Indiana Univ. Math. J. 49 (2000), no. 3, 1125-1153.

12. Claus Gerhardt, Hypersurfaces of prescribed scalar curvature in Lorentzian manifolds, J. Reine Angew. Math. 554 (2003), 157-199.

13. David Gilbarg and Neil S. Trudinger, Elliptic partial differential equations of second order, Classics in Mathematics, Springer-Verlag, Berlin, 2001.

14. Bo Guan, The Dirichlet problem for Monge-Ampère equations in non-convex domains and spacelike hypersurfaces of constant Gauss curvature, Trans. Amer. Math. Soc. 350 (1998), no. 12, 4955-4971.

15. Bo Guan, Huai-Yu Jian, and Richard M. Schoen, Entire spacelike hypersurfaces of prescribed Gauss curvature in Minkowski space, J. Reine Angew. Math. 595 (2006), $167-188$.

16. Jun-ichi Hano and Katsumi Nomizu, On isometric immersions of hyperbolic plane into the Lorentz-Minkowski space and the Monge-Ampère equation of a certain type, Math. Ann. 262 (1983), no. 1, 245-253.

17. An Min Li, Spacelike hypersurfaces with constant Gauss-Kronecker curvature in the Minkowski space, Arch. Math. 64 (1995), no. 1, 534-551.

18. Oliver C. Schnürer and Hartmut R. Schwetlick, Translating solutions for Gauß curvature flows with Neumann boundary conditions, Pacific J. Math. 213 (2004), no. 1, 89-109.

19. Wan-Xiong Shi, Deforming the metric on complete Riemannian manifolds, J. Differential Geom. 30 (1989), no. 1, 223-301.

20. Andrejs E. Treibergs, Entire spacelike hypersurfaces of constant mean curvature in Minkowski space, Invent. Math. 66 (1982), no. 1, 39-56.

21. Neil S. Trudinger, On the Dirichlet problem for Hessian equations, Acta Math. 175 (1995), no. 2, 151-164. 
HYPERSURFACES OF CONSTANT GAUSS CURVATURE IN MINKOWSKI SPACE 31

Pierre Bayard, Instituto de Física y Matemáticas. U.M.S.N.H. Ciudad UniverSitaria. CP. 58040 Morelia, Michoacán, Mexico

E-mail address: bayard@ifm.umich.mx

Oliver Schnürer, Freie Universität Berlin, Arnimallee 6, 14195 Berlin, GerMANY

E-mail address: Oliver.Schnuerer@math.fu-berlin.de 